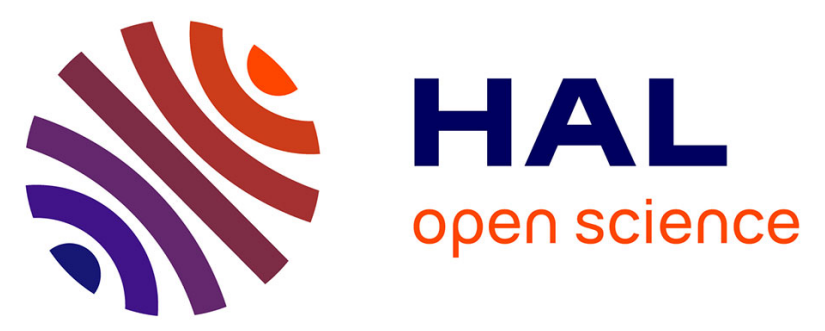

\title{
Diffracting-grain identification from electron backscatter diffraction maps during residual stress measurements: a comparison between the $\sin 2 \psi$ and $\cos \alpha$ methods
} Dorian Delbergue, Damien Texier, Martin Lévesque, Philippe Bocher

\section{- To cite this version:}

Dorian Delbergue, Damien Texier, Martin Lévesque, Philippe Bocher. Diffracting-grain identification from electron backscatter diffraction maps during residual stress measurements: a comparison between the $\sin 2 \psi$ and $\cos \alpha$ methods. Journal of Applied Crystallography, 2019, 52 (4), pp.828-843. 10.1107/S1600576719008744 . hal-02199848

\section{HAL Id: hal-02199848 \\ https://imt-mines-albi.hal.science/hal-02199848}

Submitted on 31 Jul 2019

HAL is a multi-disciplinary open access archive for the deposit and dissemination of scientific research documents, whether they are published or not. The documents may come from teaching and research institutions in France or abroad, or from public or private research centers.
L'archive ouverte pluridisciplinaire HAL, est destinée au dépôt et à la diffusion de documents scientifiques de niveau recherche, publiés ou non, émanant des établissements d'enseignement et de recherche français ou étrangers, des laboratoires publics ou privés. 


\title{
Diffracting-grain identification from electron backscatter diffraction maps during residual stress measurements: a comparison between the $\sin ^{2} \psi$ and $\cos a$ methods
}

\author{
Dorian Delbergue, ${ }^{\mathrm{a}, \mathrm{b}}$ Damien Texier, ${ }^{\mathrm{c}}$ Martin Levesque ${ }^{\mathrm{b}}$ and Philippe Bocher $^{\mathrm{a} *}$ \\ åDepartment of Mechanical Engineering, École de Technologie Supérieure, 1100 Rue Notre-Dame Ouest, Qc H3C 1K3, \\ Montréal, Canada, 'b Department of Mechanical Engineering, École Polytechnique de Montréal, Qc H3T 1J4, Montréal, \\ Canada, and ${ }^{\mathrm{c}}$ Institut Clément Ader (ICA), Université de Toulouse, CNRS, IMT Mines Albi, INSA, ISAE-SUPAERO, UPS, \\ Campus Jarlard, F-81013 Albi, France. *Correspondence e-mail: philippe.bocher@etsmtl.ca
}

\begin{abstract}
$\mathrm{X}$-ray diffraction (XRD) is a widely used technique to evaluate residual stresses in crystalline materials. Several XRD measurement methods are available. (i) The $\sin ^{2} \psi$ method, a multiple-exposure technique, uses linear detectors to capture intercepts of the Debye-Scherrer rings, losing the major portion of the diffracting signal. (ii) The $\cos \alpha$ method, thanks to the development of compact 2D detectors allowing the entire Debye-Scherrer ring to be captured in a single exposure, is an alternative method for residual stress measurement. The present article compares the two calculation methods in a new manner, by looking at the possible measurement errors related to each method. To this end, sets of grains in diffraction condition were first identified from electron backscatter diffraction (EBSD) mapping of Inconel 718 samples for each XRD calculation method and its associated detector, as each method provides different sets owing to the detector geometry or to the method specificities (such as tilt-angle number or Debye-Scherrer ring division). The X-ray elastic constant (XEC) $\frac{1}{2} S_{2}$, calculated from EBSD maps for the $\{311\}$ lattice planes, was determined and compared for the different sets of diffracting grains. It was observed that the $2 \mathrm{D}$ detector captures 1.5 times more grains in a single exposure (one tilt angle) than the linear detectors for nine tilt angles. Different XEC mean values were found for the sets of grains from the two XRD techniques/detectors. Grain-size effects were simulated, as well as detector oscillations to overcome them. A bimodal grain-size distribution effect and 'artificial' textures introduced by XRD measurement techniques are also discussed.
\end{abstract}

Keywords: X-ray diffraction; residual stress;

XRD method comparison; diffracting-grain

identification; Debye rings; Inconel 718.

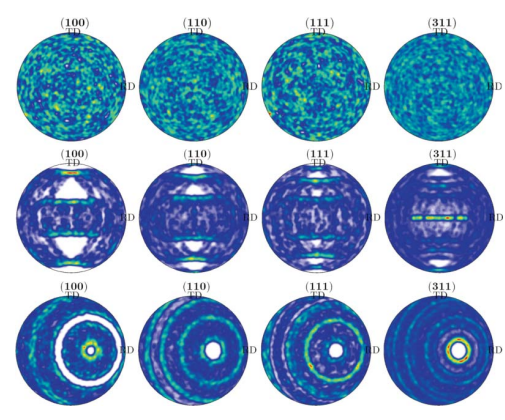

\section{Introduction}

Residual stresses play a major role in the fatigue-life behavior of mechanical components (Webster \& Ezeilo, 2001; Klotz, Delbergue et al., 2018). Residual stress states resulting from manufacturing or surface treatments are now accounted for in fatigue-life prediction models (Klotz, Miao et al., 2018). Accurate and reliable experimentally measured residual stress profiles are also required for validating predictive models of manufacturing-induced residual stresses (Guagliano, 2001; Frija et al., 2006; Gariépy et al., 2013; Heydari Astaraee et al., 2017; Tu et al., 2017, 2018).

A large number of residual stress measurement techniques exist (Schajer, 2013). The X-ray diffraction (XRD) technique is one of them and has been used for decades, to the extent that some diffractometers are specifically designed for residual stress measurement purposes (Ruud, 2002) and have become 
relatively easy to operate. Industry relies intensively on XRD for quality control of fatigue-critical components.

$\mathrm{X}$-rays are electromagnetic radiation whose wavelength is close to crystal lattice spacing. This specific feature can be exploited to measure lattice spacings (Cullity, 1956), $d$, and a constitutive theory can then be used to compute residual stresses, under the assumption of an elastic crystal lattice distortion (Prevéy, 1986). The technique relies on Bragg's condition, or the condition for diffraction, defined as

$$
n \lambda=2 d \sin \theta,
$$

where $n$ is a positive integer usually taken as equal to $1, \lambda$ is the wavelength of the radiation and $\theta$ is the Bragg angle. The Bragg condition is satisfied when the incident and diffracted $\mathrm{X}$-ray beams are bisected by the normal to the $(h k l)$ diffraction planes. For isotropic polycrystalline materials, the scattered X-rays form a cone, called the Debye-Scherrer cone (Howard, 1982). The Debye-Scherrer ring, also named Debye ring, is formed when the diffraction cone intersects a planar detector placed perpendicularly to the incoming X-ray beam (Cullity, 1956; Hörz \& Quaide, 1973). A diffraction peak is formed along a given ring radius on the detector. The peak's relative position on the detector allows determination of the Bragg angle value, and its width can be related to the cold working degree within the irradiated volume (Prevéy, 2000).

Two methods are now mainly used for X-ray residual stress calculation: the $\sin ^{2} \psi$ and the $\cos \alpha$ methods. The standardized $\sin ^{2} \psi$ method (SAE International, 2003; ASTM International, 2012) is traditionally employed in industry (Fitzpatrick et al., 2005). This method uses part of the Debye ring since the XRD is captured by two linear detectors, such as position-sensitive scintillation detectors, having a fixed position relative to the incident X-ray beam. Several measurements have to be taken at different tilt angles (Noyan \& Cohen, 1987), thus sampling different sets of grains. The residual stress associated with the measurements is obtained by linear regression from the slope of the linear relationship existing between the lattice spacings measured at different tilt angles and $\sin ^{2} \psi$ values (calculated from the tilt angles).

Technology improvements led to 2D detectors (He, 2009), such as image-plate (IP) detectors (He, 2009; Eatough et al., 1997), facilitating the use of the $\cos \alpha$ method (Taira et al., 1978; Sasaki et al., 1997). The $\cos \alpha$ method relies on the whole Debye ring, which is captured in a single exposure with an IP detector (Hiratsuka et al., 2003) having the incident X-ray beam emanating from its center. The ring's radius is then used to compute the lattice deformation and the corresponding residual stress value.

The $\sin ^{2} \psi$ and $\cos \alpha$ methods rely on different sets of detected diffracting grains owing to the difference in detector geometry, which may result in different residual stress measurements. Peterson et al. (2017) and others (Sasaki, 2014; Delbergue et al., 2016; Kohri et al., 2016; Ramirez-Rico et al., 2016; Delbergue et al., 2017) have reported differences in results when measuring residual stresses with the two methods. However, Peterson et al. (2017) were able to reduce the differences between the two methods in shot-peened standard-sample residual stress measurements with the generalized least-squares analysis proposed by Miyazaki \& Sasaki (2016). Miyazaki \& Sasaki (2016) have shown, on the basis of the fundamental equations, that the only differences between the $\sin ^{2} \psi$ and $\cos \alpha$ methods lie in the fact that the sets of diffracting grains are different for these two methods and the crystalline grain aggregates exhibit local elastic property variations (Kocks et al., 1998).

Authors who compared the residual stress measurements resulting from the $\sin ^{2} \psi$ and $\cos \alpha$ methods have mostly focused on experimental observations (Delbergue et al., 2016; Kohri et al., 2016; Ramirez-Rico et al., 2016; Peterson et al., 2017) or on the fundamental equations (Ramirez-Rico et al., 2016; Miyazaki \& Sasaki, 2016), but none have numerically compared the two methods using the coupling between the stress calculation method and the inhomogeneous grain response to elastic deformation. By relying on crystal plasticity, Erinosho et al. (2016) have simulated lattice strains by considering grains that satisfy the diffraction condition and compared their predictions against in situ synchrotron XRD measurements.

The principal objective of this article is to compare the $\sin ^{2} \psi$ and $\cos \alpha$ methods in terms of diffracting-grain number and X-ray elastic constant (XEC) of the diffracting-grain set, according to the detector specificities. The comparison was made by identifying the diffracting grains for the two methods from electron backscatter diffraction (EBSD) grain orientation maps and evaluating their average elastic properties. To the best of our knowledge, the comparison of the two methods has never been done using the XEC estimated for sets of diffracting grains from EBSD maps. The article is organized as follows: The fundamental equations for the $\sin ^{2} \psi$ and $\cos \alpha$ methods are presented in Section 2. In Section 3, the studied material, its microstructure, the two XRD measurement conditions, the grain conditions for diffraction and the XEC calculation method are presented. Experimental and numerical results such as EBSD maps, identified diffracting grains, calculated XEC and 'artificial' textures of the XRD measurement methods are set out in Section 4. The results are then discussed in Section 5 before the conclusion of this work.

\section{Residual stress measurement theory}

For crystalline or polycrystalline materials, the measurement of residual stresses using X-ray diffraction techniques is based on the change of lattice spacing, $d$, and each grain is basically used as a local strain gauge. For instance, the presence of tensile residual stresses within the irradiated volume increases the $d$ spacing in a given direction, resulting in a diffraction peak shift towards lower $\theta$ values. The shift yields a Bragg angle value, $\theta$, which differs from the $\theta_{0}$ value obtained for a stress-free sample of the same material (usually measured on powder).

In XRD measurements, the strain, $\varepsilon_{\varphi \psi}^{\{h k l\}}$, is measured along the diffraction vector $\mathbf{V}_{h k l}$ defined in the sample coordinate system as 


$$
\mathbf{V}_{h k l}=\left(\begin{array}{c}
v_{1} \\
v_{2} \\
v_{3}
\end{array}\right)=\left(\begin{array}{c}
\sin \psi \cos \varphi \\
\sin \psi \sin \varphi \\
\cos \psi
\end{array}\right)
$$

where $\varphi$ and $\psi$ are the azimuthal and tilt angles, respectively (the angles are defined in Fig. 1). The measured strain, $\varepsilon_{\varphi \psi}^{\{h k l\}}$, can be computed from the measured Bragg angle, $\theta$, or the planes' lattice spacing, $d_{\varphi \psi}^{\{h k l\}}$, and can be obtained by

$$
\varepsilon_{\varphi \psi}^{\{h k l\}}=\frac{d_{\varphi \psi}^{\{h k l\}}-d_{0}^{\{h k l\}}}{d_{0}^{\{h k l\}}}=-\Delta \theta \cot \theta_{0},
$$

where $\Delta \theta=\theta-\theta_{0}$ and $d_{0}$ is the $d$ spacing corresponding to the $\theta_{0}$ value, measured for a stress-free sample. The strain measured along the diffraction vector $\mathbf{V}_{h k l}$ can then be expressed in terms of the strain components $\varepsilon_{i j}$ in sample coordinates with respect to the Einstein summation convention by

$$
\varepsilon_{\varphi \psi}^{\{h k l\}}=\frac{d_{\varphi \psi}^{\{h k l\}}-d_{0}^{\{h k l\}}}{d_{0}^{\{h k l\}}}=v_{i} v_{j} \varepsilon_{i j}
$$

where $v_{i}$ and $v_{j}$ are the $\mathbf{V}_{h k l}$ components defined in equation (2). If the measured material is isotropic and homogeneous, Hooke's law can be used to relate the macroscopic stress, $\sigma$, to the strain as

$$
\varepsilon_{i j}=\frac{1+v}{E} \sigma_{i j}-\delta_{i j} \frac{v}{E} \sigma_{k k}
$$

where $E$ and $v$ are the material's macroscopic Young modulus and Poisson ratio values, respectively, and $\delta$ is the Kronecker delta. Assuming that the material is macroscopically not textured and composed of fine grains, and if the stress in the irradiated layer is assumed to be biaxial (i.e. $\sigma_{33}=\sigma_{23}=\sigma_{13}=$ 0 ), the measured strain can be related to the macroscopic stress through

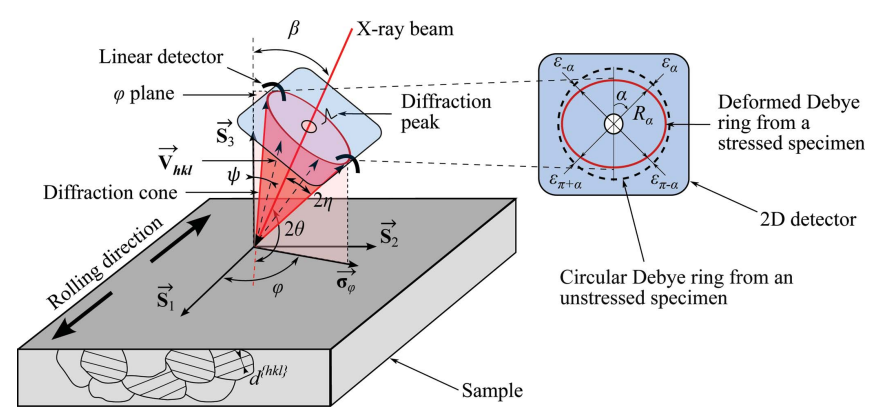

Figure 1

Schematic representation of a sample, the diffraction cone resulting from the diffraction of $\{h k l\}$ planes, and the measurement planes related to the linear and 2D detectors $\left(\sin ^{2} \psi\right.$ and $\cos \alpha$ methods, respectively). List of the symbols: $d^{[h k l\}}$ : lattice spacing of the $\{h k l\}$ planes; $\mathbf{V}_{h k l}$ : diffraction vector; $\mathbf{S}$ : sample coordinate system; $2 \theta$ : Bragg angle; $2 \eta$ : diffraction cone semi-apex; $\beta$ : incident X-ray beam angle; $\psi$ : angle between the $\{h k l\}$ plane normal and sample normal (tilt angle); $\varphi$ : azimuthal angle; $\boldsymbol{\sigma}_{\varphi}$ : residual stress measurement direction; $\alpha: 2 \mathrm{D}$ detector angle; $R_{\alpha}$ : Debye ring radius for a given $\alpha ; \varepsilon_{\alpha}$ : strain measured for a given $\alpha$. $\varepsilon_{\varphi \psi}^{\{h k l\}}=\frac{d_{\varphi \psi}^{\{h k l\}}-d_{0}^{\{h k l\}}}{d_{0}^{\{h k l\}}}=\frac{1+v^{\{h k l\}}}{E^{\{h k l\}}} \sigma_{\varphi} \sin ^{2} \psi-\frac{\nu^{\{h k l\}}}{E^{\{h k l\}}}\left(\sigma_{11}+\sigma_{22}\right)$,

where $E^{\{h k l\}}$ and $v^{\{h k l\}}$ are the equivalent Young modulus and Poisson ratio determined for the $\{h k l\}$ planes, respectively. $\sigma_{\varphi}=\sigma_{11} \cos ^{2} \varphi+\sigma_{12} \sin 2 \varphi+\sigma_{22} \sin ^{2} \varphi$ is the macroscopic stress along the direction $\varphi$ defined by the linear detector plane (see Fig. 1). The use of $E^{\{h k l\}}$ and $v^{\{h k l\}}$ allows the crystal anisotropy to be taken into consideration to relate the strain measured on crystallographic planes to the macroscopic stress. We define $S_{1}^{\{h k l\}}$ and $\frac{1}{2} S_{2}^{\{h k l\}}$ as the XECs for the $\{h k l\}$ planes as

$$
\begin{gathered}
S_{1}^{\{h k l\}}=\frac{-v^{\{h k l\}}}{E^{\{h k l\}}}, \\
\frac{1}{2} S_{2}^{\{h k l\}}=\frac{1+v^{\{h k l\}}}{E^{\{h k l\}}} .
\end{gathered}
$$

The stress $\sigma_{\varphi}$ can be obtained by differentiating equation (6) with respect to $\sin ^{2} \psi$ as

$$
\sigma_{\varphi}=\frac{E^{\{h k l\}}}{1+v^{\{h k l\}}} \frac{\partial \varepsilon_{\varphi \psi}^{\{h k l\}}}{\partial \sin ^{2}}=\frac{1}{\frac{1}{2} S_{2}^{\{h k l\}}} \frac{\partial \varepsilon_{\varphi \psi}^{\{h k l\}}}{\partial \sin ^{2}},
$$

which shows that only $\frac{1}{2} S_{2}^{\{h k l\}}$ has to be known to determine $\sigma_{\varphi}$. When plotting the measured strain $\varepsilon_{\varphi \psi}^{\{h k l\}}$ as a function of $\sin ^{2} \psi$, and on the basis of the assumptions listed previously, one can determine the stress $\sigma_{\varphi}$, in the measurement direction $\varphi$, if a linear regression between $\varepsilon_{\varphi \psi}^{\{h k l\}}$ and $\sin ^{2} \psi$ is performed. Therefore, several $\psi$ angles have to be used to obtain the relationship between $\varepsilon_{\varphi \psi}^{\{h k l\}}$ and $\sin ^{2} \psi$ when the diffracted $\mathrm{X}$-rays are captured with two linear detectors. In practice, the several $\psi$ angles needed, which correspond to different $\mathbf{V}_{h k l}$ vectors, are obtained when changing the incident $\mathrm{X}$-ray beam angle, $\beta=\psi \pm \eta(2 \eta=\pi-2 \theta$ being the diffraction cone semiapex angle shown in Fig. 1).

The stress calculation can be done with a single X-ray exposure when the whole Debye ring is measured. For a stress-free material, such as a powder, the Debye ring is perfectly circular, as the lattice spacings, $d_{0}^{\{h k l\}}$, are the same in every direction. A stressed surface will modify the ring radius and its distortion can be described using the $\alpha$ angle defined as the polar angle along the Debye ring, as schematized in Fig. 1. The strain is measured along $\mathbf{V}_{h k l}$ for different $\alpha$ angles. Therefore, when the measurement is made with a planar detector, the diffraction vector $\mathbf{V}_{h k l}$ should be defined in the sample coordinate system using the angle $\alpha$ and the diffraction cone's semi-apex angle $2 \eta$ as

$$
\begin{aligned}
& \mathbf{V}_{h k l}= \\
& \left(\begin{array}{c}
\cos \eta \sin \beta \cos \varphi-\sin \eta \cos \beta \cos \varphi \cos \alpha-\sin \eta \sin \varphi \sin \alpha \\
\cos \eta \sin \beta \sin \varphi-\sin \eta \cos \beta \sin \varphi \cos \alpha+\sin \eta \cos \varphi \sin \alpha \\
\cos \eta \cos \beta+\sin \eta \sin \beta \cos \alpha
\end{array}\right) .
\end{aligned}
$$


For a given $\alpha$-angle position on the 2D detector, the measured strain $\varepsilon_{\alpha}^{\{h k l\}}$ can be obtained from the ring radius $R_{\alpha}$ as

$$
\varepsilon_{\alpha}^{\{h k l\}}=-\Delta \theta \cot \theta_{0}=\frac{1}{2}\left[2 \theta_{0}-\pi+\tan ^{-1}\left(R_{\alpha} / C_{L}\right)\right] \cot \theta_{0},
$$

where $C_{L}$ is the sample-to-detector distance. Similarly to equation (4), the measured strain $\varepsilon_{\alpha}^{\{h k l\}}$ can be related to the strain components $\varepsilon_{i j}$ in sample coordinates with respect to the Einstein summation convention as

$$
\varepsilon_{\alpha}^{\{h k l\}}=v_{i} v_{j} \varepsilon_{i j} .
$$

We define the parameter $\bar{\varepsilon}_{\alpha}^{\{h k l\}}$ as

$$
\bar{\varepsilon}_{\alpha}^{\{h k l\}}=\frac{1}{2}\left[\left(\varepsilon_{\alpha}^{\{h k l\}}-\varepsilon_{\pi+\alpha}^{\{h k l\}}\right)+\left(\varepsilon_{-\alpha}^{\{h k l\}}-\varepsilon_{\pi-\alpha}^{\{h k l\}}\right)\right],
$$

where $\varepsilon_{\alpha}^{\{h k l\}}, \varepsilon_{\pi+\alpha}^{\{h k l\}}, \varepsilon_{-\alpha}^{\{h k l\}}$ and $\varepsilon_{\pi-\alpha}^{\{h k l\}}$ are strains determined at four points located at $90^{\circ}$ on the Debye ring for a given $\alpha$ angle varying from 0 to $90^{\circ}$ using equation (10). The $\cos \alpha$ method yields the stress $\sigma_{\varphi}$ thanks to some trigonometric simplifications. When a biaxial stress state is assumed in the irradiated layer and if the material's microstructure is composed of fine grains with a macroscopically isotropic elastic behavior, the parameter $\bar{\varepsilon}_{\alpha}^{\{h k l\}}$ can be expressed in terms of stresses by combining equations (5), (11), (9) and (12) as

$$
\bar{\varepsilon}_{\alpha}^{\{h k l\}}=-\frac{1+v^{\{h k l\}}}{E^{\{h k l\}}} \sin 2 \eta \sin 2 \beta \cos \alpha \sigma_{\varphi},
$$

where $\sigma_{\varphi}=1 / 2\left[\sigma_{11}(1+\cos 2 \varphi)+\sigma_{22}(1-\cos 2 \varphi)+2 \sigma_{12} \sin 2 \varphi\right]$. Differentiating equation (13) with respect to $\cos \alpha$ and isolating $\sigma_{\varphi}$ yields

$$
\begin{aligned}
\sigma_{\varphi} & =-\frac{E^{\{h k l\}}}{1+v^{\{h k l\}}} \frac{1}{\sin 2 \eta \sin 2 \beta} \frac{\partial \bar{\varepsilon}_{\alpha}^{\{h k l\}}}{\partial \cos \alpha} \\
& =-\frac{1}{\frac{1}{2} S_{2}^{\{h k l\}}} \frac{1}{\sin 2 \eta \sin 2 \beta} \frac{\partial \bar{\varepsilon}_{\alpha}^{\{h k l\}}}{\partial \cos \alpha} .
\end{aligned}
$$

The stress $\sigma_{\varphi}$ can be obtained when plotting the parameter $\bar{\varepsilon}_{\alpha}^{\{h k l\}}$ as a function of $\cos \alpha$ and if a linear regression between $\bar{\varepsilon}_{\alpha}^{\{h k l\}}$ and $\cos \alpha$ is performed.

\section{Material and experimental procedures}

\subsection{Material}

This study focused on the nickel-base superalloy Inconel 718 (IN718), which was subjected to a solution and precipitation heat treatment as per AMS 5663M (SAE International, 2009). The material's chemical composition and macro-tensile properties were determined in previous work (Klotz, Delbergue et al., 2018) and are listed in Tables 1 and 2, respectively. Two samples extracted in the rolling direction of bars with initial diameters of $25.4 \mathrm{~mm}$ (1 in) and $76.2 \mathrm{~mm}$ (3.5 in) were investigated. As they provide different microstructure, the bars' initial diameters in inches will be used to refer to the samples. The sample surfaces were carefully prepared by grinding using $\mathrm{SiC}$ papers (down to grade 1200/ $\mathrm{P} 4000$ ), and a layer of $5 \mu \mathrm{m}$ was then removed by electropolishing to obtain surfaces that were almost free of residual stress and work hardening.
Table 1

Inconel 718 chemical composition obtained by optical spectrometry (wt \%).

\begin{tabular}{lllllllllll}
\hline Elements & $\mathrm{Ni}$ & $\mathrm{Fe}$ & $\mathrm{Cr}$ & $\mathrm{Nb}$ & $\mathrm{Mo}$ & $\mathrm{Ti}$ & $\mathrm{Al}$ & $\mathrm{Co}$ & $\mathrm{Mn}$ & $\mathrm{Si}$ \\
\hline Composition & Balance & 19.53 & 17.84 & 5.02 & 3.07 & 1.16 & 0.64 & 0.35 & 0.16 & 0.06 \\
\hline
\end{tabular}

Table 2

Inconel 718 macrotensile properties.

$E$ : Young's modulus; $\sigma_{\mathrm{y} 0.2 \%}: 0.2 \%$ offset yield strength; $\sigma_{\mathrm{u}}$ : ultimate strength; El.: elongation at failure.

\begin{tabular}{llll}
\hline$E(\mathrm{GPa})$ & $\sigma_{y 0.2 \%}(\mathrm{MPa})$ & $\sigma_{\mathrm{u}}(\mathrm{MPa})$ & El. $(\%)$
\end{tabular}

Table 3

Typical X-ray diffraction conditions for Proto iXRD $\left(\sin ^{2} \psi\right.$ method) and Pulstec $\mu-\mathrm{X} 360 \mathrm{n}(\cos \alpha$ method) apparatuses.

\begin{tabular}{lll}
\hline & $\begin{array}{l}\text { Proto } \\
\text { iXRD }\end{array}$ & $\begin{array}{l}\text { Pulstec } \\
\mu-X 360 n\end{array}$ \\
\hline Tube (X-ray lines) & Mn $K \alpha$ & $\mathrm{Cr} K \beta$ \\
Wavelength $(\AA)$ & 2.291 & 2.085 \\
Bragg angle $2 \theta_{0}\left(^{\circ}\right)$ & 151.8 & 148.2 \\
Diffraction planes $/ d_{0}^{\{h k l\}}$ & & $\{311\} / d_{0}^{\{311\}}=1.08 \AA$ \\
Collimator (mm) & 1 & 1 \\
Voltage $(\mathrm{kV}) / \mathrm{current}(\mathrm{mA})$ & $23 / 2.5$ & $30 / 1.0$ \\
Total measurement time $(\mathrm{min})$ & 8 & 1.5 \\
No. of inclinations & 9 & 1 \\
$\beta$ inclinations $\left(^{\circ}\right)$ & {$[ \pm 25 \pm 19.01 \pm 14.06 \pm 7.270]$} & 30 \\
Sample-to-detector distance $(\mathrm{mm})$ & 39.5 & 38.55 \\
\hline
\end{tabular}

The nickel-base superalloy being a face-centered cubic (f.c.c.) polycrystalline material, the $\{311\}$ family of atom planes is used since they diffract for high $2 \theta$ Bragg angles, reducing the sensitivity of the strain to the precision of the Bragg angle measurement. In this condition, 24 planes of symmetry are available, meaning that 24 planes can possibly contribute to the diffraction of X-rays.

\subsection{Diffractometers for XRD measurements}

We consider two dedicated diffractometers, one for each stress calculation method, that could be used for residual stress measurements. A Proto iXRD apparatus equipped with two linear detectors (position-sensitive scintillation detectors) and an Mn tube could be used to investigate the $\sin ^{2} \psi$ method, and a Pulstec $\mu$-X360n apparatus equipped with a 2D detector (IP detector) and a Cr tube could be used when capturing the whole Debye ring for the $\cos \alpha$ method. The Proto iXRD Mn tube allows measurement of the deformation of the IN718 $\{311\}$ planes from filtered $K \alpha$ lines, whereas use of the Pulstec $\mu$-X360n Cr tube implies measuring it from the $K \beta$ line. The $K \alpha$ and $K \beta$ lines are explained in standard reference books (Cullity, 1956; Noyan \& Cohen, 1987). Typical diffraction conditions for IN718 and for both apparatuses are listed in Table 3.

\subsection{Microstructural characterization method}

EBSD scans were performed to characterize the grain-size distribution, quantify the degree of crystallographic texture and investigate the effect of the set of grains participating in 
Table 4

EBSD subset map size and total map area for 1 and 3.5 in samples.

\begin{tabular}{lll}
\hline Sample & Subset map size $(\mu \mathrm{m})$ & Total map area $\left(\mathrm{mm}^{2}\right)$ \\
\hline 1 in & $317 \times 237.5$ & 1.414 \\
3.5 in & $635 \times 476$ & 1.154 \\
\hline
\end{tabular}

the XRD measurements on the XEC values. The scans were carried out on areas having sizes similar to the irradiated areas during XRD measurements $\left(\sim 2 \mathrm{~mm}^{2}\right)$. A conventional Hitachi SU-70 scanning electron microscope equipped with a Bruker camera and HKL Channel 5 software from Oxford Instruments were used for the EBSD scans. Table 4 summarizes the subset and total map areas measured with a step size of $0.5 \mu \mathrm{m}$.

Grain-size distributions and the crystallographic texture were determined by post-treatment using MATLAB (The MathWorks Inc., Natick, MA, USA) and the MTEX opensource package (Bachmann et al., 2011). Grains were identified using a grain-detection angle of $10^{\circ}$. Twins were considered as unique grains to limit the artifacts arising from the determination of the grain's mean orientation, thus increasing the total number of indexed grains. The orientation distribution functions (ODFs) were calculated using the de la Vallée Poussin kernel (Hielscher, 2013) and all measurement points to account for grain-size effects from surface observation. Sample texture analyses are presented as inverse pole figure plots with respect to the rolling direction. Texture analyses associated with XRD measurements are also presented using pole figures.

\subsection{Identification of diffracting grains}

A MATLAB script using the $M T E X$ open-source package has been developed to identify the grains meeting the Bragg condition for the two types of detector. Under this condition and for an unstressed sample, the diffracting planes have to make an angle $\theta_{0}$ with the incoming X-rays to scatter the incoming X-ray beam for the particular planes under consideration, i.e. $\{311\}$, as shown in Fig. 2. In this figure, $\mathbf{V}_{\mathrm{IXR}}, \mathbf{V}_{\mathrm{DXR}}$

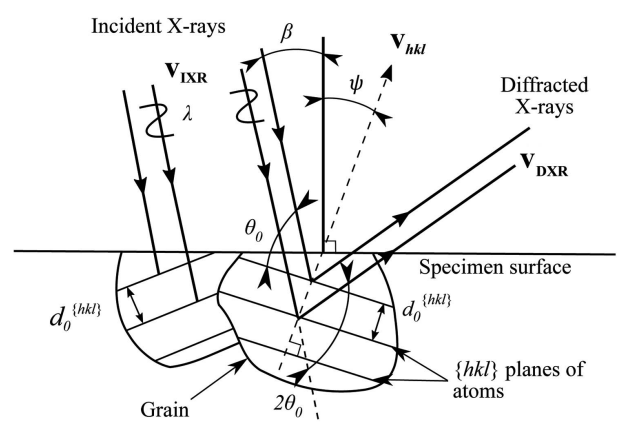

Figure 2

Illustration of X-rays diffracted by $\{h k l\}$ planes of an unstressed grain in the Bragg condition. In this condition, the diffraction vector $\mathbf{V}_{h k l}$ bisects the incident X-rays $\mathbf{V}_{\text {IXR }}$ and the diffracted X-rays $\mathbf{V}_{\text {DXR }}$, such that they make an angle $\theta_{0}$ with the $\{h k l\}$ planes having a lattice spacing $d_{0}^{\{h k l\}}$. List of symbols: $\beta$ : incident X-ray beam angle; $\psi$ : angle between the $\{h k l\}$ plane normal and sample normal (tilt angle); $\lambda$ : X-ray wavelength. and $\mathbf{V}_{h k l}$ are unit vectors representing the incident $\mathrm{X}$-rays, the diffracted X-rays and the vector normal to the $\{h k l\}$ planes, respectively. To meet the conditions of Bragg's law, $\mathbf{V}_{h k l}$ has to be the bisector vector of $\mathbf{V}_{\text {IXR }}$ and $\mathbf{V}_{\text {DXR }}$, providing a geometrical condition for selecting the diffracting grains. The $M T E X$ package was used for calculating each grain's mean orientation. The $\mathbf{V}_{h k l}$ vector can thus be determined for any ( $h$, $k, l)$ Miller indices and the condition for diffraction can be expressed by the dot product of $\mathbf{V}_{\text {IXR }}$ and $\mathbf{V}_{h k l}$. Each grain can be associated with an angle $\theta_{x}$ defined as

$$
\theta_{x}=\cos ^{-1}\left(\frac{\mathbf{V}_{\mathrm{IXR}} \cdot \mathbf{V}_{h k l}}{\left\|\mathbf{V}_{\mathrm{IXR}}\right\|\left\|\mathbf{V}_{h k l}\right\|}\right)
$$

and diffraction can take place when $\theta_{x}=\left(90-\theta_{0}\right)$. A tolerance of $2^{\circ}$ was used to replicate the XRD line-broadening effect, allowing some deviation from the exact Bragg condition. The $2^{\circ}$ tolerance is close to half the diffraction-peak widths experimentally obtained for both techniques in previous work (Delbergue et al., 2017; Klotz, Delbergue et al., 2018), focusing the investigation on the diffraction information coming from the highest intensities of potential diffraction peaks.

\subsection{XEC calculation}

The XEC $\frac{1}{2} S_{2}^{\{h k l\}}$ of the diffracting grains was determined for comparison purposes using the MTEX package (Mainprice et al., 2011) as follows:

(i) An IN718 single-crystal stiffness tensor, $\mathbf{C}_{\mathrm{IN} 718}$, was assigned to all the grains of the EBSD map: $C_{11}=251.0 \mathrm{GPa}$, $C_{12}=135.5 \mathrm{GPa}$ and $C_{44}=98.0 \mathrm{GPa}$. The coefficients have been chosen from among the experimental values reported in the literature (Ledbetter \& Reed, 1973; Margetan et al., 2005; Haldipur, 2006; Aba-Perea et al., 2016) at room temperature for a certain range of grain sizes and compositions.

(ii) An ODF, $f$, was calculated for the set of diffracting grains to account for the possibility of some anisotropy introduced by the XRD measurement.

(iii) The ODF was then used to determine the average stiffness tensor of the set of diffracting grains computed for the Voigt and Reuss bounds (assuming a constant elastic strain and stress, respectively, in all crystallites) as (Mainprice et al., 2011)

$$
\begin{gathered}
\langle\mathbf{C}\rangle^{\text {SVoigt }}=\sum_{m=1}^{M} \mathbf{C}_{\mathrm{IN} 718}\left(g_{m}\right) \omega_{m} f\left(g_{m}\right), \\
\langle\mathbf{C}\rangle^{\text {SReuss }}=\left[\sum_{m=1}^{M} \mathbf{C}_{\mathrm{IN} 718}^{-1}\left(g_{m}\right) \omega_{m} f\left(g_{m}\right)\right]^{-1},
\end{gathered}
$$

where $\langle\mathbf{C}\rangle^{\text {SVoigt }}$ and $\langle\mathbf{C}\rangle^{\text {SReuss }}$ are the average stiffness tensors expressed in the sample reference system, $\mathrm{S}$, for the Voigt and Reuss bounds, respectively, and are calculated for the selected set of $m$ diffracting grains having an orientation $g_{m}$ and a weight $\omega_{m}$. The angle brackets denote the average over the grains that satisfy a particular diffraction condition.

(iv) The XEC $\frac{1}{2} S_{2}^{\{h k l\}}$ was calculated with the quasi-isotropic XEC equation used in the case of biaxial stress state (Van Houtte \& De Buyser, 1993): 


$$
\frac{1}{2} S_{2}^{\{h k l\}}=\left\langle S_{3333}\right\rangle^{\mathrm{L}}-\left\langle S_{3311}\right\rangle^{\mathrm{L}},
$$

where $\left\langle S_{3333}\right\rangle^{\mathrm{L}}$ and $\left\langle S_{3311}\right\rangle^{\mathrm{L}}$ are the fourthrank compliance tensor coefficients of $\left\langle\left.\mathbf{S}_{i j k l}\right|^{\mathrm{L}}=\mathbf{a}_{i m} \mathbf{a}_{j n} \mathbf{a}_{k o} \mathbf{a}_{l p}\left\langle\mathbf{S}_{\text {mnop }}\right\rangle^{\mathrm{S}}\right.$ expressed in the laboratory system, L, with $\left\langle\mathbf{S}_{i j k l}\right\rangle^{\mathrm{S}}=\left(\left\langle\mathbf{C}_{i j k l}\right\rangle^{\mathrm{S}}\right)^{-1}$. The laboratory system is defined as having the $z$ axis coincident with the diffraction vector, and $a_{i j}$ is the rotation matrix from the $\mathrm{S}$ system to the $\mathrm{L}$ system. $\frac{1}{2} S_{2}^{\{h k l\}}$ was computed for the Voigt and Reuss bounds.

(v) Finally, as the experimentally measured elastic constants are often close to the average of the Voigt and Reuss bounds (Hill, 1952; Gnäupel-Herold et al., 1998; Murray, 2013), the Neerfeld-Hill limit, which is the arithmetic mean of the Voigt and Reuss bounds, was used during the study:

$$
\left(\frac{1}{2} S_{2}^{\{h k l\}}\right)^{\text {Hill }}=\frac{1}{2}\left[\left(\frac{1}{2} S_{2}^{\{h k l\}}\right)^{\text {Voigt }}+\left(\frac{1}{2} S_{2}^{\{h k l\}}\right)^{\text {Reuss }}\right] .
$$

Note that the $\langle h k l\rangle$ crystallographic direction is taken into consideration in the calculation of $f\left(g_{m}\right)$ as it is computed for the identified diffracting grains. When computed for the $\{311\}$ planes, the $\operatorname{XEC}\left(\frac{1}{2} S_{2}^{\{111\}}\right)^{\text {Hill }}$ is denoted in this article as $\frac{1}{2} S_{2}^{\{311\}}$ for convenience.

This procedure for the $\frac{1}{2} S_{2}^{\{311\}}$ computation was carried out for each of the different $\beta$ tilts and linear detectors for the $\sin ^{2} \psi$ method and for each combination of $\beta$ tilt and $\alpha$-angle position for the $\cos \alpha$ method.

\section{Results \\ 4.1. Microstructure analyses}

The grain microstructure was characterized by EBSD over regions representative of the X-ray irradiated zones. Large areas in the RD-TD plane were scanned for both 1 and 3.5 in samples. The resulting maps were plotted for the RD using inverse pole figures (IPFs) and are presented in Fig. 3 (RD: rolling direction; TD: transverse direction; ND: normal direction). Each color corresponds to a crystal orientation. The 1 in sample has a fine and homogeneous grain-size distribution, while the 3.5 in sample exhibits a bimodal microstructure composed of small grains and a few large grains. The grains' equivalent diameter distributions are presented as a function of the area they occupy in Fig. 4. A $6 \mu \mathrm{m}$ average grain size was calculated for the 35805 detected grains for the 1 in sample, whereas the average grain size was $8 \mu \mathrm{m}$ for the 3.5 in sample and 10610 grains were detected [corresponding to an ASTM grain-size number of 12 and 11 (ASTM International, 2013), respectively]. Table 5 summarizes the grain distribution for the two specimens. The 3.5 in sample's bimodal microstructure is documented in Fig. 4(b) (inset), where the grains are divided into two families: the 'large grains' were defined as the grains larger than the average grain size plus four times the equivalent diameter standard deviation (SD), i.e. an equivalent diameter

higher than $42 \mu \mathrm{m}$ [the equivalent diameter, $\emptyset$, is calculated as $\emptyset=2(A / \pi)^{1 / 2}$, where $A$ is the grain area]. Ninety-three large grains ( $0.87 \%$ of the grain population) were found, occupying $26 \%$ of the map area.

The crystallographic textures are presented in Fig. 5 as IPF plots with respect to the RD, for both samples. No preferred

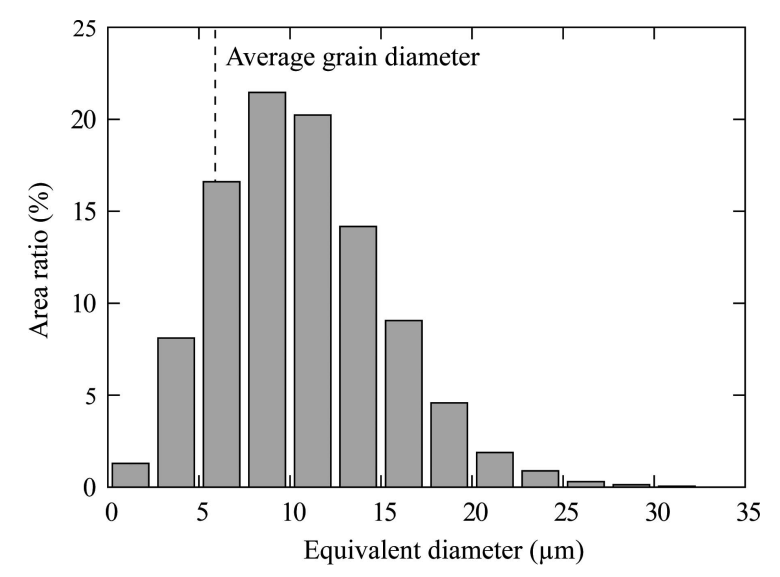

(a)

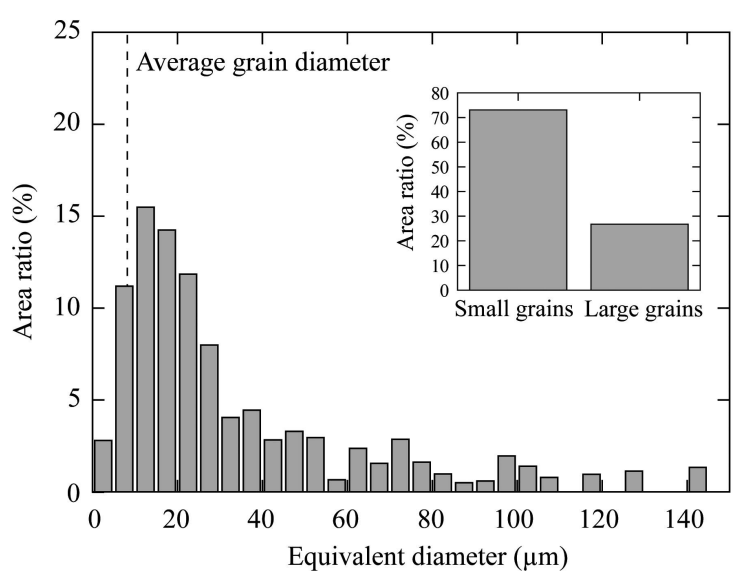

Figure 4

(b)

Grain-size distribution in percent of area ratio for $(a)$ the 1 in sample and (b) the 3.5 in sample. The equivalent diameter, $\emptyset$, is calculated as $\emptyset=2(A$ / $\pi)^{1 / 2}$, where $A$ is the grain area. In the inset, 'large grains' describes the grains having a diameter of at least the average diameter plus four times the diameter standard deviation $(\emptyset>42 \mu \mathrm{m})$. 
Table 5

Grain size and grain density for 1 and 3.5 in samples.

\begin{tabular}{llllll}
\hline \multicolumn{6}{c}{ Grain equivalent diameter $(\mu \mathrm{m})$} \\
\hline Sample & Mean & SD & Minimum & Maximum & Grain density $\left(\right.$ grains mm $\left.^{-2}\right)$ \\
\hline 1 in & 6 & 4 & 1 & 31 & 25316 \\
3.5 in & 8 & 8 & 1 & 140 & 9200 \\
\hline
\end{tabular}

orientation is observed for the 1 in sample with a texture index of 1.15 , whereas for the 3.5 in sample, a slightly higher occurrence of the $\langle 001\rangle$ crystallographic orientations is found in the RD, but with a low index of around 1.9. Such a texture is often observed after recrystallization of a rolled f.c.c. material (Etter \& Baudin, 2013). Nevertheless, neither sample can be considered as highly textured. The crystallographic directions of interest $\langle 311\rangle$ are also shown in Fig. 5.

\subsection{Diffracting grains}

4.2.1. Identification of the diffracting grains. The grains in the diffraction condition were first identified using the developed MATLAB script on the 1 in sample, as it represents the

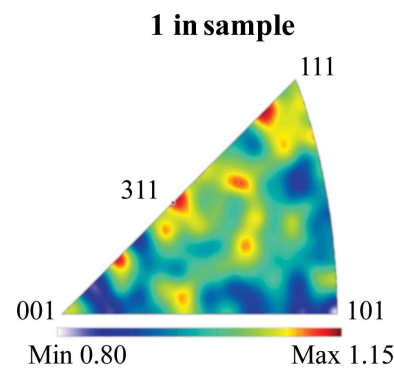

(a)

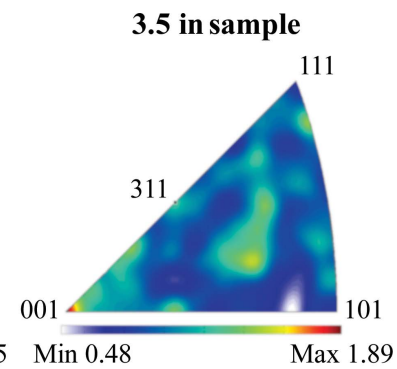

(b)
Figure 5

Inverse pole figures for the texture analysis of the $(a) 1$ in and $(b) 3.5$ in samples with respect to the rolling direction. The $\langle 311\rangle$ directions are also shown.

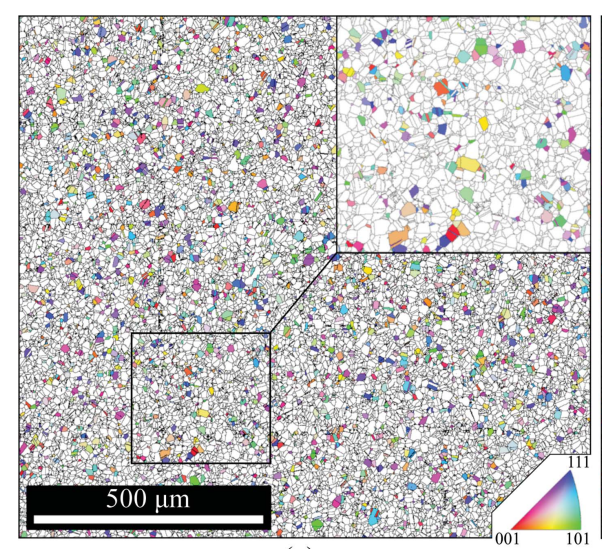

(a)

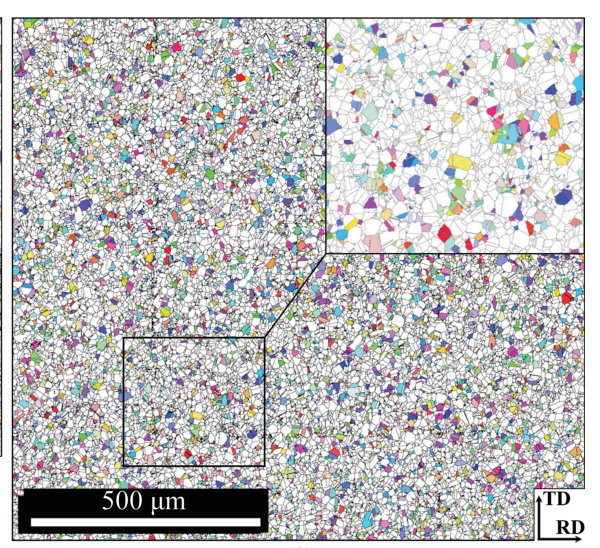

(b)
Figure 6

Orientation distribution maps of the 1 in sample presenting the diffracting grains detected by $(a)$ the two linear detectors for nine inclinations (Proto iXRD) and $(b)$ the 2D detector for a single exposure (Pulstec $\mu$-X360n). The IPF representation with respect to the RD has been kept.
Number of grains contributing to the XRD when data are captured by two linear detectors or a $2 \mathrm{D}$ detector for the 1 in sample.

\begin{tabular}{lll}
\hline & Proto iXRD & Pulstec $\mu$-X360n \\
\hline Detector type & Linear detectors & 2D detector \\
No. of diffracting grains & 5540 & 8183 \\
Grain percentage (\%) & 15.5 & 22.9 \\
Area percentage $(\%)$ & 15.0 & 22.4 \\
\hline
\end{tabular}

ideal case of a homogeneous microstructure. The XRD conditions and diffractometer parameters used in the script are listed in Table 3. The maximum effective penetration depth, when computed for the two radiations and detector types, as described in the work of Noyan \& Cohen (1987) and Tanaka (2018) for the $\sin ^{2} \psi$ and $\cos \alpha$ methods, respectively, was found to be $5 \mu \mathrm{m}$, which is less than the smaller average grain size $(6 \mu \mathrm{m})$. Therefore, it is assumed that only the layer of grains obtained by EBSD could diffract.

Figs. $6(a)$ and $6(b)$ present in IPF coloring the diffracting grains if X-ray diffraction was captured by the two linear detectors of the Proto iXRD and the 2D detector of the Pulstec $\mu-X 360$ n, respectively. Fig. 6(a) is the superimposition of the diffracting grains captured by the two linear detectors for nine acquisition positions (the nine $\beta$ angles listed in Table 3$)$. Fig. $6(b)$ shows the diffracting grains seen by the $2 \mathrm{D}$ detector for a single exposure at $\beta=30^{\circ}$. The number of diffracting grains was calculated in the two situations and is reported in Table 6 . The grains contributing to the diffraction data represent 15.0 and $22.4 \%$ of the EBSD map area for the $\sin ^{2} \psi$ and $\cos \alpha$ methods, respectively. The 2D detector thus samples $32 \%$ more unique diffracting grains than the linear detectors swept along nine $\beta$ angles. Only 1129 unique grains are identified both by the two linear detectors (5540 identified diffracting grains) and by the 2D detector (8183 identified diffracting grains), corresponding to 20 and $14 \%$, respectively. Some of these shared diffracting grains can be observed in Fig. 6 .

4.2.2. Simulation of detector windows. The intersections of the diffraction cone with the linear and $2 \mathrm{D}$ detectors were simulated, to better understand how the $2 \mathrm{D}$ detector can see $32 \%$ more diffracting grains in a single exposure than the two linear detectors in nine exposures. Figs. 7(a) and 7(b) present the simulated images seen by the two linear detectors at $\beta=25^{\circ}$ and by the $2 \mathrm{D}$ detector at $\beta=30^{\circ}$, respectively, for a single exposure. Each circular symbol represents the localization of X-rays scattered by a diffracting grain intersecting the detector plane (as illustrated in Fig. 1). The diffracting grains' mean orientations are presented using IPF coloring with respect to the RD. The $X$ axis is parallel to the RD while the $Y$ axis is 
parallel to the TD, and the incoming X-rays (not represented here) would be at the coordinates $(0,0)$. The two linear detectors can be observed in Fig. 7( $a$ ) with the presence of two distinct regions. The full Debye ring is captured by the 2D detector as shown in Fig. 7(b). Note that the $Y$ width of the regions in Fig. $7(a)$ is related to the width of the linear detectors, whereas the $X$ width is linked to the $2^{\circ}$ tolerance angle used for the identification of the diffracting grains, which also corresponds to the ring width in Fig. 7(b).

\subsection{Angle variations}

4.3.1. Linear detectors: number of used $\beta$ angles. Three to $21 \beta$ angles have been simulated for the same range of maximum angles: $\pm 25^{\circ}$. Increasing the number of $\beta$ angles would increase not only the number of diffracting grains but also the measurement time. Fig. 8 presents the number of unique diffracting grains versus the number of $\beta$ angles. The values are compared with the number of unique diffracting grains seen by the $2 \mathrm{D}$ detector for a measurement at $\beta=30^{\circ}$ [the grains highlighted in Fig. 6(b)]. The case of nine $\beta$ angles corresponds to the number of measurements at which the

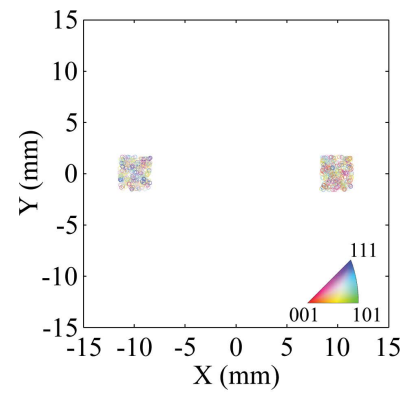

(a)

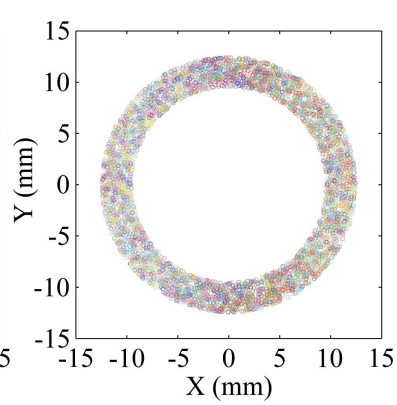

(b)
Figure 7

Simulated images seen by $(a)$ the two linear detectors $\left(\beta=25^{\circ}\right)$ and $(b)$ the $2 \mathrm{D}$ detector $\left(\beta=30^{\circ}\right)$ for the 1 in sample. Diffracting grains are represented by circular symbols, and IPF coloring with respect to the RD is used to show their mean orientations.

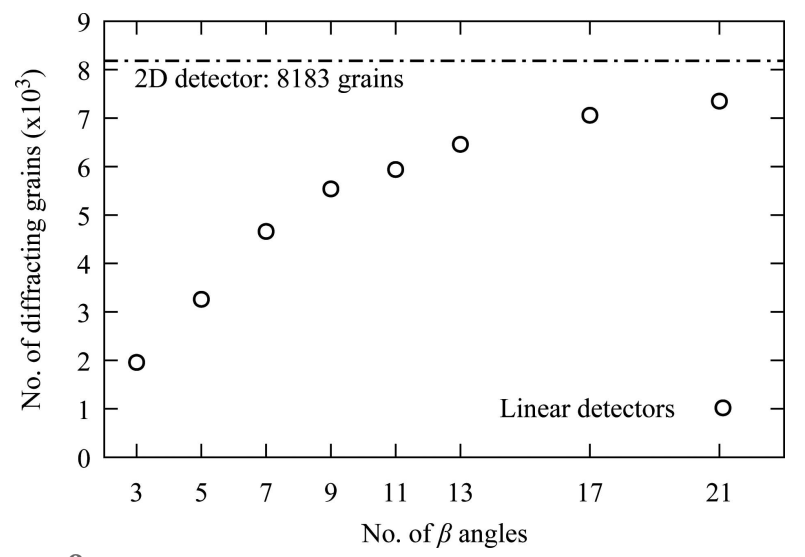

Figure 8

Total number of unique diffracting grains versus the number of $\beta$ angles taken by the two linear detectors in a $\pm 25^{\circ}$ range. The number of unique diffracting grains contributing to the $2 \mathrm{D}$ detector data for a single exposure at $\beta=30^{\circ}$ is plotted as a dash-dot line for comparison. number of additional diffracting grains starts to decrease with increasing number of $\beta$ angles.

The total number of diffracting grains stays below the 8183 unique grains contributing to the diffraction captured by the $2 \mathrm{D}$ detector, even for a high number of linear-detector positions (e.g. $21 \beta$ angles). Increasing the number of $\beta$ angles further than 21 does not increase the number of unique diffracting grains owing to the superimposition of the $\beta$ positions.

4.3.2. 2D detector: effect of $\beta$ angle. The influence of the $\mathrm{X}$-ray incident angle on the number of diffracting grains has been studied for the $\cos \alpha$ method. $\beta$ angles ranging from 10 to $45^{\circ}$ have been simulated. Fig. 9 presents the number of unique diffracting grains for the different 2D detector inclinations. The figure shows that the number of unique diffracting grains remains almost constant, regardless of the $\beta$ angle. This observation might result from the fact that the 1 in sample exhibits an isotropic distribution of the grains' mean orientations.

4.3.3. 2D detector: variation along the $a$ values. The parameter $\bar{\varepsilon}_{\alpha}^{\{h k l\}}$ is computed, for a given $\alpha$ angle, from the strains measured at four orthogonal positions on the Debye

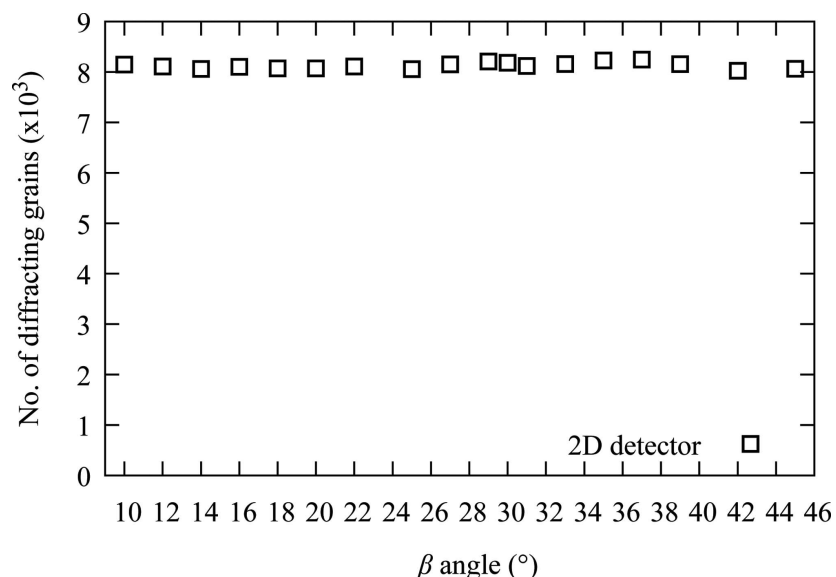

Figure 9

$\beta$ angle $\left({ }^{\circ}\right)$

Total number of unique diffracting grains seen by the $2 \mathrm{D}$ detector versus the detector $\beta$ angle.

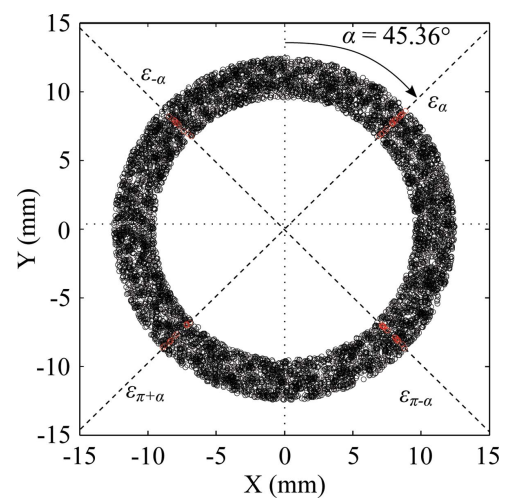

Figure 10

Simulated Debye ring on the $2 \mathrm{D}$ detector for $\beta=30^{\circ}$. Each circle represents a diffracting grain. The diffracting grains used for the calculation of $\bar{\varepsilon}_{\alpha}^{\{h k l\}}$ at $\alpha=45.36^{\circ}$ are plotted in red as an example. 
ring. Consequently, the full scan of the Debye ring is carried out for a variation of $\alpha$ from 0 to $90^{\circ}$. Therefore, only a small part of the collected data is used for computing the strain for a given $\alpha$. Fig. 10 presents a simulated Debye ring seen on a $2 \mathrm{D}$ detector for a single exposure at $\beta=30^{\circ}$. Each circle corresponds to a given diffracting grain. The grains used for the calculation of $\bar{\varepsilon}_{\alpha}^{\{h k l\}}$ at $\alpha=45.36^{\circ}$ are highlighted in red as an example. The $\alpha$ step size being set to $0.72^{\circ}, 125 \alpha$ angles are used to compute the $125 \bar{\varepsilon}_{\alpha}^{\{h k l\}}$ values. The $\bar{\varepsilon}_{\alpha}^{\{h k l\}}$ values may then be plotted versus the corresponding $\cos \alpha$ values to determine the residual stress. In the case where no diffracting grains are detected for a given $\alpha$, the $\alpha$-step size could be increased to allow $\bar{\varepsilon}_{\alpha}^{\{h k l\}}$ values to be computed.

\subsection{XRD texture measurement}

Different sets of diffracting grains are detected when using the two diffraction techniques, and different crystallographic textures may be obtained via the linear detectors and the 2D detector during XRD measurements. Using the developed script and the MTEX package, pole figures can be plotted for the homogeneous microstructure of the 1 in bar, showing the diffracting grains for the two different types of detectors. The pole figures for the (100), (110), (111) and (311) sets of planes are presented in Figs. 11(a), 11(b) and 11(c) for all the grains of the 1 in sample, and only for the diffracting grains detected by the two linear detectors and the 2D detector, respectively. The (311) pole figure has been plotted in addition to the traditional (100), (110) and (111) pole figures to present the textures introduced by XRD measurements of the planes used for strain measurements.

Like Fig. 6(a), Fig. $11(b)$ is the superimposition of the different positions taken by the linear detectors. However, Figs. $12(a), 12(b)$ and $12(c)$ show the pole figures for three specific $\beta$ angles taken by the linear detectors: $\beta=-25^{\circ}, \beta=0^{\circ}$ and $\beta=25^{\circ}$, respectively. For each pole figure, two hot spots representing the localization of the two linear detectors can be observed. They are the centers of concentric circles. The detector motion during the residual stress measurements can be observed as a straight line at the center of the (311) pole figure in Fig. 11(b), which results from the displacement of the concentric circles, illustrated in Fig. 12. On the other hand, the 2D detector, by its geometry, allows the Debye ring to be captured in its entirety, which results in the circle of high index visible in the (311) pole figure of Fig. 11(c). The concentric circles are not centered because of the incident angle of the $\mathrm{X}$-ray beam $\left(\beta=30^{\circ}\right)$. Nonetheless, the $\cos \alpha$ method implies dividing the Debye ring for the $\bar{\varepsilon}_{\alpha}^{\{h k l\}}$ calculation, as shown in computation.
Fig. 10, which results in the intermediate textures presented in Fig. 13. The $\alpha$ steps providing the highest and the lowest texture indexes are presented in Figs. 13(a) and 13(b),

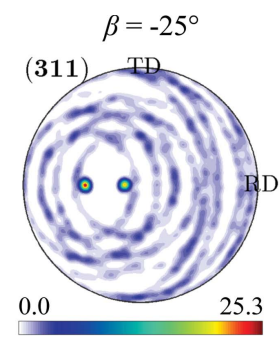

(a)

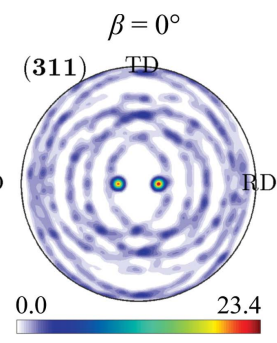

(b)
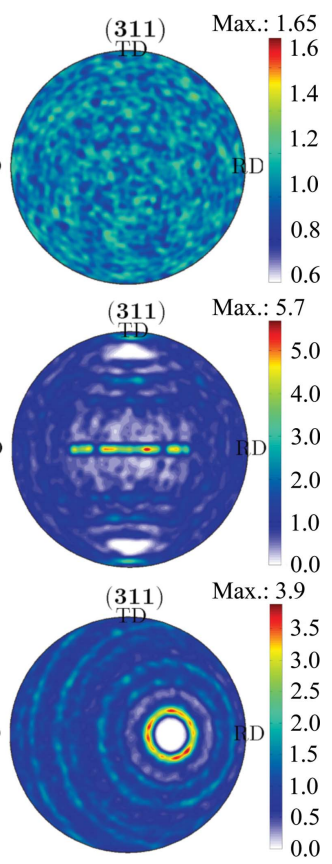

0.0 65
.6
.4
.2
.0
.8
.6
.0
.0
.0
.0
.0
.0 3.9 .5 .0
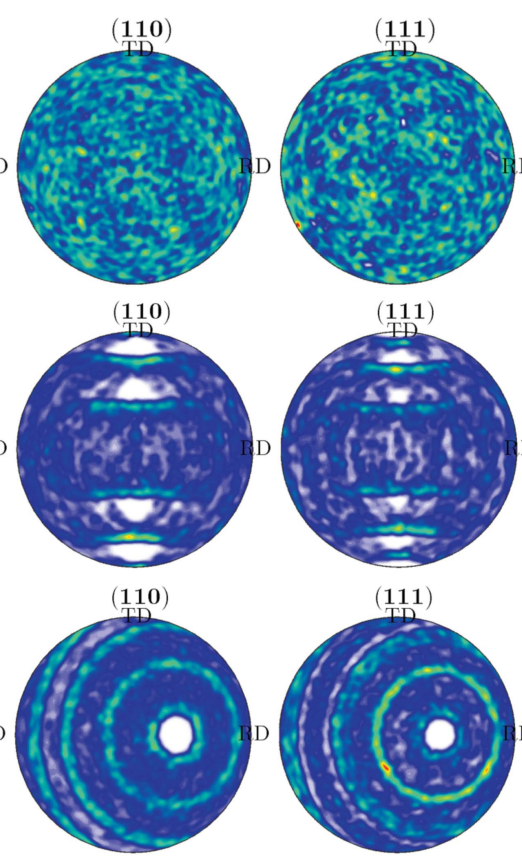

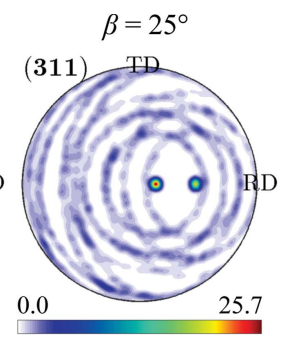

(c) (1)

(1) Pole figures of the (100), (110), (111) and (311) sets of planes for $(a)$ the whole 1 in sample EBSD map and for the diffracting grains detected by $(b)$ the linear detectors and $(c)$ the 2D detector. Note that the (311) set of planes is the strain measurement set of planes used for residual stress

Figure 12

Pole figures of the (311) diffracting planes for the two linear detectors at (a) $\beta=-25^{\circ}$, (b) $\beta=0^{\circ}$ and $(c) \beta=25^{\circ}$.

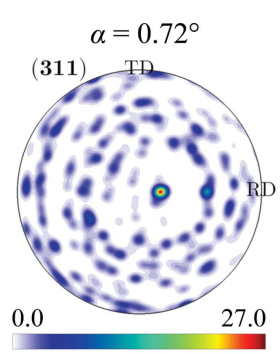

(a)

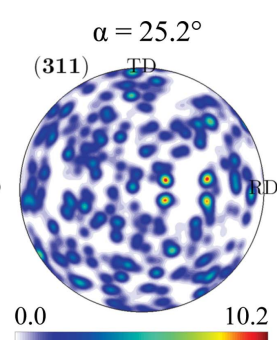

(b)

Figure 13

Pole figures of the (311) diffracting planes for the 2D detector for the $\alpha$ angles providing the maximum and minimum texture indexes at $(a) \alpha=$ $0.72^{\circ}$ and $(b) \alpha=25.2^{\circ}$, respectively, as well as the $\alpha=45.36^{\circ}$ example. 
respectively. Fig. 13(c) corresponds to the pole figure of the highlighted grains given as an example in Fig. 10. The four strain measurements at $\alpha, \pi+\alpha,-\alpha$ and $\pi-\alpha$ imply four hot spots [visible in Figs. 13(b) and 13(c)], the centers of four concentric circles, but because of the low $\alpha$-step size the circles are not clearly defined. Furthermore, for $\alpha$ values close to $0^{\circ}$ or to $90^{\circ}$, only two hot spots can be distinguished, which results in higher maximum texture indexes.

Fig. 11(a) confirms that the 1 in sample is not textured with a maximum index of 1.65 , while the pole figures of the diffracting grains detected by the two types of detectors [Figs. 11(b), 11(c), 12 and 13] exhibit two different types of textures. These textures are related to the type of detector used for XRD measurements. This 'artificial' texture may affect the XEC value of the diffracting grains and therefore the measured stress, as the stress is linearly dependent on the $\mathrm{XEC}$ value in both calculation methods.

\subsection{XEC values for the diffracting grains}

The average XEC $\frac{1}{2} S_{2}^{\{h k l\}}$ was determined for the $\{311\}$ planes of the diffracting grains in terms of the Hill limit. As described in Section 3.5, ODFs were used for XEC calculations to take into account the 'artificial' texture created by XRD conditions.

The $\frac{1}{2} S_{2}^{\{h k l\}}$ Voigt and Reuss bounds can be computed as a reference for the case of an isotropic polycrystal and for the stiffness tensor given in Section 3.5 as (Van Houtte \& De Buyser, 1993)

$$
\begin{gathered}
\left(\frac{1}{2} S_{2}^{\{h k l\}}\right)^{\text {Voigt }}=\frac{10 S_{1212}\left(S_{1111}-S_{1122}\right)}{3 S_{1111}-3 S_{1122}+4 S_{1212}} \\
\left(\frac{1}{2} S_{2}^{\{h k l\}}\right)^{\text {Reuss }}=S_{1111}-S_{1122}-3 S_{0} \Gamma
\end{gathered}
$$

where $S_{1111}, S_{1212}$ and $S_{1122}$ are the coefficients of the singlecrystal compliance tensor $\left[\mathbf{S}_{\mathrm{IN718}}=\left(\mathbf{C}_{\mathrm{IN} 718}\right)^{-1}\right], S_{0}=S_{1111}-$ $S_{1122}-2 S_{1212}$, and $\Gamma$ is the orientation parameter defined as

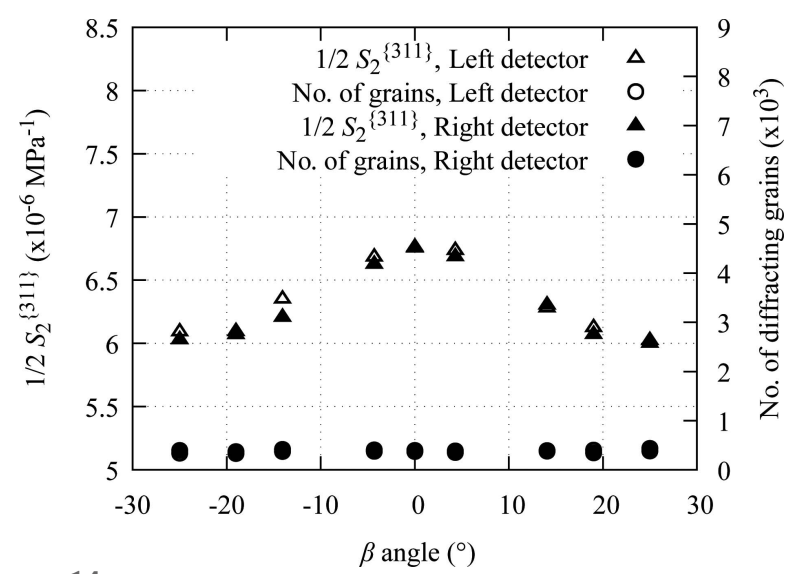

Figure 14

Number of diffracting grains detected by the two linear detectors for nine $\beta$ angles and their corresponding XEC $\frac{1}{2} S_{2}^{\{311\}}$ values calculated for the $\{311\}$ planes.

$$
\Gamma=\frac{h^{2} k^{2}+h^{2} l^{2}+k^{2} l^{2}}{\left(h^{2}+k^{2}+l^{2}\right)^{2}} .
$$

Only the Reuss bound is $h k l$ dependent. When computed for the case of the $\{311\}$ planes, the Voigt and Reuss bounds are $6.11 \times 10^{-6}$ and $6.98 \times 10^{-6} \mathrm{MPa}^{-1}$, respectively. Finally, the Hill limit computed using equation (18) is $6.54 \times 10^{-6} \mathrm{MPa}^{-1}$.

Fig. 14 presents the XEC values for the nine $\beta$ angles used for residual stress measurements using the linear detectors as well as the number of diffracting grains detected for each position. The results are separately given for the two linear detectors, namely the left and right detectors [they correspond in Fig. 7(a) to the circles in negative and positive $X$ values, respectively, or to the left and right concentric circles in Fig. 12]. The number of diffracting grains remains almost constant, around 380 diffracting grains per detector and per inclination. For the nine positions and the two linear detectors, the XEC average and confidence interval, $\mathrm{CI}_{95 \%}$, are $6.33 \times 10^{-6}$ and $0.23 \times 10^{-6} \mathrm{MPa}^{-1}$, respectively. The $\mathrm{CI}_{95 \%}$ value is computed from the lower bound of the $95 \%$

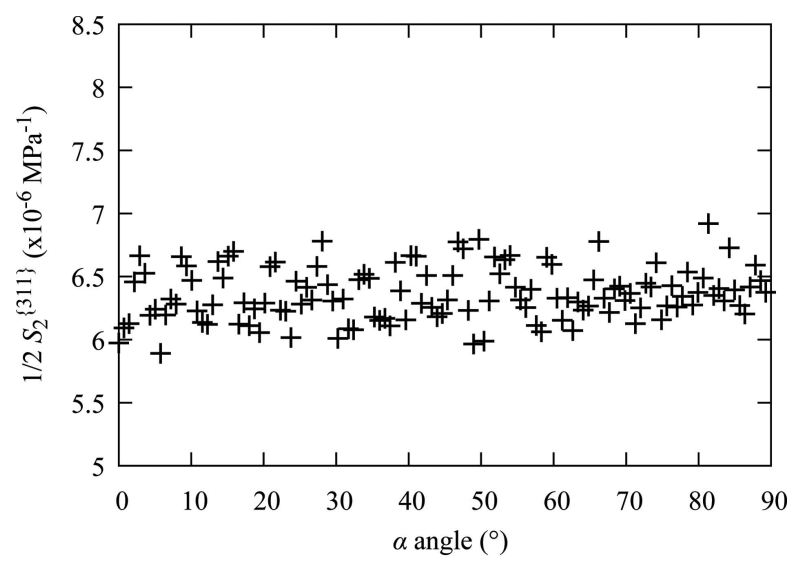

(a)

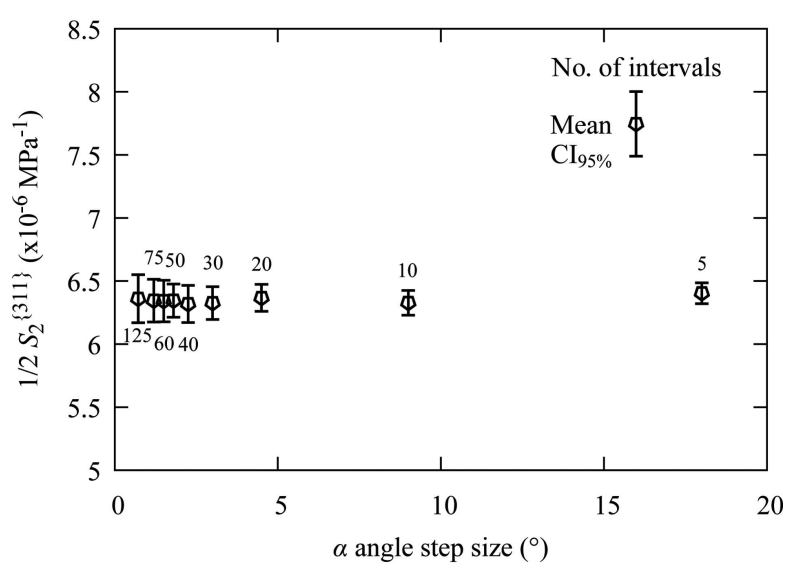

(b)

Figure 15

(a) XEC $\frac{1}{2} S_{2}^{\{311\}}$ values calculated for the diffracting grains detected by the 2D detector at $\beta=30^{\circ}$ for $125 \alpha$ angles (from 0 to $90^{\circ}$ ). (b) Mean and confidence interval $\left(\mathrm{CI}_{95 \%}\right)$ of the XEC $\frac{1}{2} S_{2}^{\{111\}}$ values calculated for different $\alpha$-step sizes. The corresponding number of $\alpha$ angles is presented below or above the error bar. $\mathrm{CI}_{95 \%}$ is the lower bound of the $95 \%$ confidence interval on the standard deviation. 


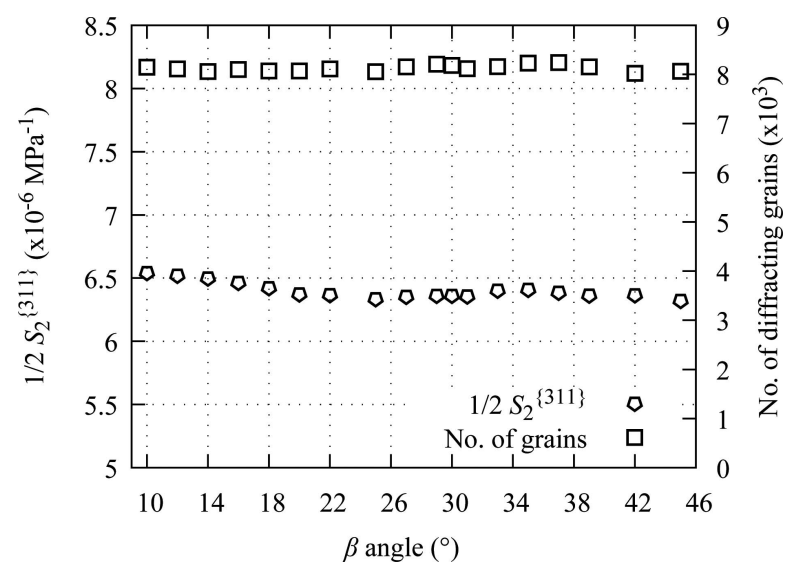

Figure 16

Number of diffracting grains detected by the 2D detector for different $\beta$ angles and corresponding XEC $\frac{1}{2} S_{2}^{\{311\}}$ values calculated for a $0.72^{\circ} \alpha$-step size (125 $\alpha$ angles).

confidence interval on the standard deviation of an assumed normal distribution, determined with a $\chi^{2}$ test (Hines et al., 2008). Thus, in this case, the XEC standard deviation is less than $0.23 \times 10^{-6} \mathrm{MPa}^{-1}$ at the $95 \%$ level of confidence.

For the $2 \mathrm{D}$ detector, the $\frac{1}{2} S_{2}^{\{311\}}$ values were calculated for a $0.72^{\circ} \alpha$-step size to characterize the ring geometry. The 125 XEC values determined for $\beta=30^{\circ}$ are presented in Fig. 15(a). The values range from $5.89 \times 10^{-6}$ to $6.92 \times 10^{-6} \mathrm{MPa}^{-1}$ and a mean value of $6.36 \times 10^{-6} \mathrm{MPa}^{-1}$ is observed, yielding a $\mathrm{CI}_{95 \%}$ of $0.19 \times 10^{-6} \mathrm{MPa}^{-1}$. This low $\alpha$-step size implies five times fewer diffracting grains per $\alpha$ value than for a given $\beta$ position with the two linear detectors, resulting in a larger range of the computed values but a lower $\mathrm{CI}_{95 \%}$ value (as the confidence interval is also based on the population size). Fig. 15(b) shows the mean and the confidence interval values of the XEC calculated for different $\alpha$-step sizes. It can be observed that increasing the step size narrows the confidence interval $\mathrm{CI}_{95 \%}$, while the mean value remains almost constant. Indeed, decreasing the number of intervals increases the number of orientations accounted for in one interval, which results in better approximation of the XEC.

Different $\beta$ angles have also been simulated for the 2D detector and $0.72^{\circ} \alpha$-step size. The average XEC value and the number of diffracting grains for the different $\beta$ angles are shown in Fig. 16. A quasi-constant number of diffracting grains can be observed throughout the entire range of simulated $\beta$ angles. The average XEC value slightly decreases for high $\beta$ angles (a $0^{\circ} \beta$ angle corresponds to a normal incident $\mathrm{X}$-ray beam).

For the linear detectors, the average XEC value of the diffracting grains is higher than the random texture XEC value, whereas for the $2 \mathrm{D}$ detector it is slightly lower. These differences are most likely to be due to the fact that the XEC is determined on sets of grains having a slight texture. The XEC values computed for the two detector types are not

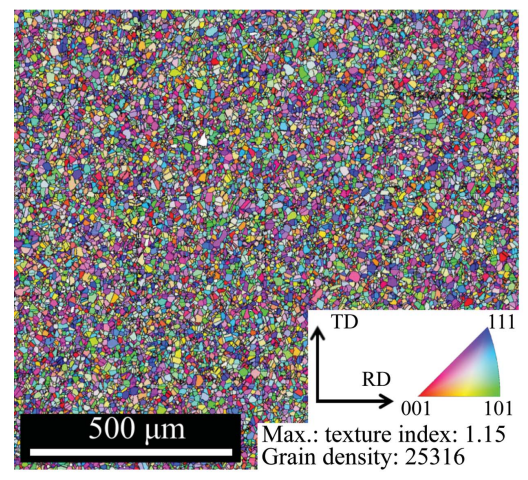

(a)

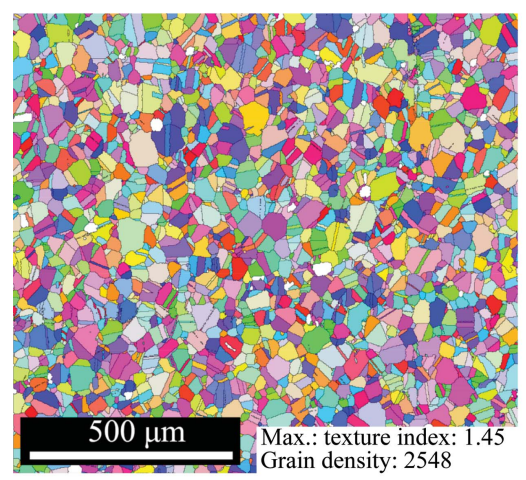

(d)

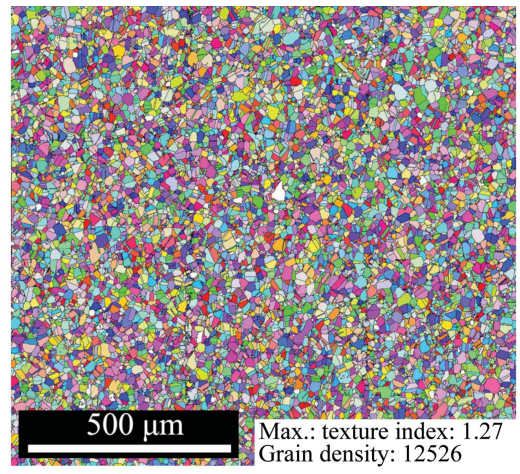

(b)

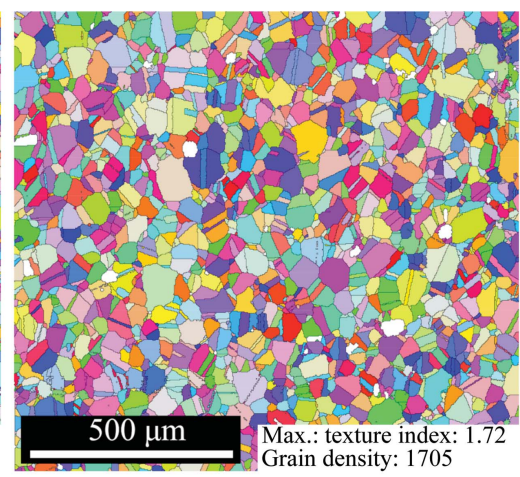

(e)

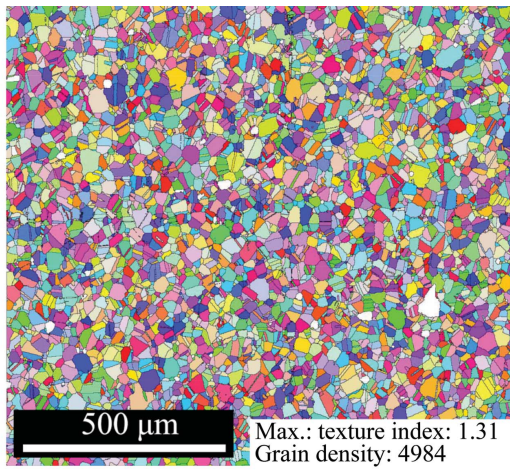

(c)

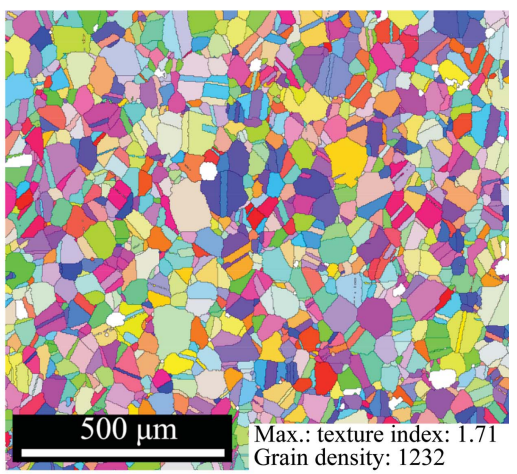

$(f)$

Figure 17

Orientation distribution maps of 1 in sample for the following reduction factors: $(a) R=1$ (initial map), $(b) R=1 / 2,(c) R=1 / 5,(d) R=1 / 10,(e) R=1 / 15$ and $(f) R=1 / 20$. Information on map maximum texture index and grain density (in grains $\mathrm{mm}^{-2}$ ) is also provided. 
statistically different and seem not to be highly affected by the detector's position, i.e. $\beta$ angle, in the case of homogeneous microstructure.

\subsection{Effect of grain size}

XRD measurement quality may suffer when a relatively small number of orientations are diffracting, leading to anisotropy inherent to the used XRD method. The grain size or grain density can therefore affect the XRD measurement. The effect of grain size on the XEC was studied by 'artificially' reducing the grain density. The initial EBSD map size was reduced while keeping the same irradiated zone area, decreasing the grain density. Five resized EBSD maps, using reduction ratios of $R=1 / 2,1 / 5,1 / 10,1 / 15$ and $1 / 20$, were thus investigated, providing grain densities ranging from 12526 to 1232 grains $\mathrm{mm}^{-2}$. Note that the initial EBSD map grain density is 25316 grains $\mathrm{mm}^{-2}$ (Table 5), corresponding to an average grain size of $6 \mu \mathrm{m}$ (ASTM grain size number 12). A $1 / 20$ reduction ratio corresponds to a 20 times increase of the grain size, giving a $120 \mu \mathrm{m}$ average equivalent diameter (ASTM grain size number 3) or a grain density of 1232 grains $\mathrm{mm}^{-2}$.

Fig. 17 presents the different reduced maps as orientation distribution maps with respect to the RD. The map reduction was set to start from the upper-left corner of the initial EBSD map, shown in Fig. 17(a). The grain size was artificially increased by focusing the area of interest on a smaller portion of the initial map and by keeping the same scale. The IPFs have also been determined for each map and maximum texture indexes are reported in Fig. 17. It can be observed that increasing the average grain size slightly increases the maximum texture index without creating a specific texture. Fig. 18 presents, for the six cases, the total number of grains and the total number of unique diffracting grains for both detectors. The number of grains is reduced from 35805 (25 316 grains $\mathrm{mm}^{-2}$ ) to 1743 (1232 grains $\mathrm{mm}^{-2}$ ). Constant percentages of diffracting grains were found for the linear detectors and the 2D detector, of 15 and $23 \%$, respectively.

The calculated XEC was only affected by the change in grain density, owing to the homogeneous grain orientation and grain-size distributions. For the five resized maps, XEC $\frac{1}{2} S_{2}^{\{311\}}$ mean and confidence interval values were calculated for both diffractometer types and were compared with the results of the initial EBSD map $(R=1)$. The results are presented in Fig. 19. The $\frac{1}{2} S_{2}^{\{311\}}$ values increase by up to $2.1 \%$ as the grain density decreases, whereas the confidence interval significantly broadens. For the 2D detector, increasing the $\alpha$-step size increases the number of grains taken into consideration for each XEC computation, broadening the confidence interval $\mathrm{CI}_{95 \%}$. When the number of diffracting grains decreases significantly, the $\alpha$-step size for analyzing the Debye ring has to be increased to $1.2^{\circ}$ to ensure the presence of diffraction data for all $\alpha$ intervals. For instance, in the case of $R=1 / 20$, the $\frac{1}{2} S_{2}^{\{311\}}$ values can only be computed for an $\alpha$-step size of $1.2^{\circ}$ or higher. The values vary from

Figure 19

Mean values and confidence interval on the standard deviation $\left(\mathrm{CI}_{95}\right)$ of the XEC $\frac{1}{2} S_{2}^{\{311\}}$ determined for the $\{311\}$ planes of the diffracting grains detected by the linear and $2 \mathrm{D}$ detectors in the case of five resized maps. Results for the $2 \mathrm{D}$ detector are presented for 0.72 and $1.2^{\circ} \alpha$-step sizes. The $\mathrm{CI}_{95 \%}$ values are computed from the lower bounds of the $95 \%$ confidence interval on the standard deviation. 
$5.16 \times 10^{-6}$ to $8.40 \times 10^{-6} \mathrm{MPa}^{-1}$, yielding a $0.58 \times$ $10^{-6} \mathrm{MPa}^{-1}$ confidence interval. The high $\mathrm{CI}_{95 \%}$ values can still be explained by the very low number of diffracting grains contributing to each XEC estimation. Therefore, a high $\alpha$-step size should be used when large grains (or low grain density) are found in the material.

\subsection{Effect of diffractometer oscillation}

Experimentally, the number of diffracting grains can be increased by oscillating the X-ray beam in the $\varphi$ plane. Different oscillation values have been simulated around the $\beta$ position for both diffractometers using $1^{\circ}$ oscillation steps, meaning that for a $\beta=25^{\circ}$ position and a $2^{\circ}$ oscillation the $\mathrm{X}$-ray beam and the detectors were oscillating from 23 to $27^{\circ}$. The simulations were realized for the reduction ratios $R=1 / 15$ and $R=1 / 20$, corresponding to 90 and $120 \mu \mathrm{m}$ average grain sizes, respectively. The low grain density allows only a small number of diffracting grains, yielding a broader XEC confidence interval. The results are presented in Fig. 20, and the values for the steady positions (presented in Figs. 18 and 19) are included as $0^{\circ}$ oscillations. For both diffractometers, the oscillations increase the number of diffracting grains detected by the detectors. A $2^{\circ}$ oscillation captures up to $55 \%$ more diffracting grains for the 2D detector, whereas the linear detectors only capture up to $36 \%$ more grains. Increasing the
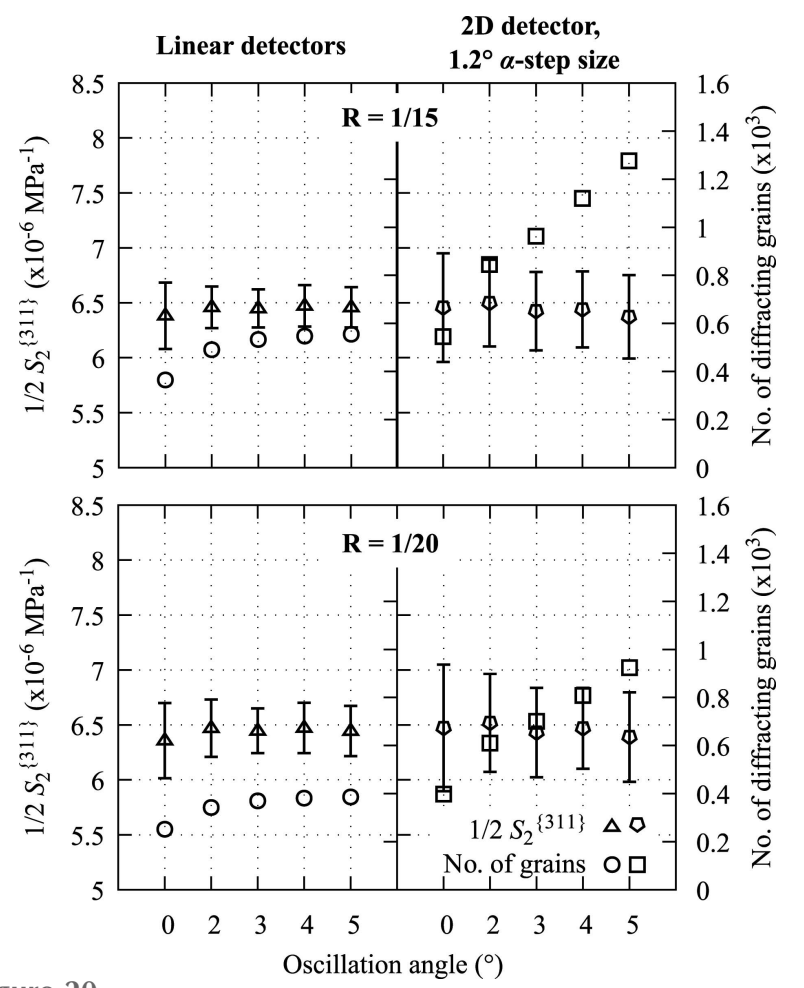

Figure 20

Total number of unique diffracting grains and XEC $\frac{1}{2} S_{2}^{\{311\}}$ mean values calculated for two different reduction factors $(R=1 / 15$ and $R=1 / 20)$ and for measurements with both techniques in the case of oscillations in the $\varphi$ plane. The calculations for the $2 \mathrm{D}$ detector were performed using $1.2^{\circ}$ $\alpha$-step sizes. The error bars represent the confidence interval on the standard deviation $\left(\mathrm{CI}_{95 \%}\right)$. oscillation angle for the linear detectors does not significantly increase the total number of diffracting grains because the oscillation for a $\beta$ position ends up superimposing the oscillations of the other $\beta$ positions, hence the asymptotic curves. The XEC values tend to decrease with the oscillations for the 2D detector, while increasing for the two linear detectors. With oscillations, the confidence interval on the standard deviation is found to be narrower by up to 43 and $36 \%$ for the linear detectors and the $2 \mathrm{D}$ detector, respectively. For the two reduction ratios, the narrowest confidence intervals are found for 3 and $4^{\circ}$ oscillations for the linear detectors and the 2D detector, respectively.

\subsection{Effect of inhomogeneous microstructure}

A bimodal microstructure composed of a large number of grains having a $6 \mu \mathrm{m}$ average grain size and very few grains with a grain size higher than $100 \mu \mathrm{m}$ was found in the 3.5 in sample, as depicted in Figs. 3 and $4(b)$. The XEC calculations for both microstructures are presented in Fig. 21(a) for the two linear detectors using nine $\beta$ angles. The average XEC and confidence interval values of the nine $\beta$ angles are $6.41 \times 10^{-6}$ and $0.33 \times 10^{-6} \mathrm{MPa}^{-1}$, respectively, for the 3.5 in sample.

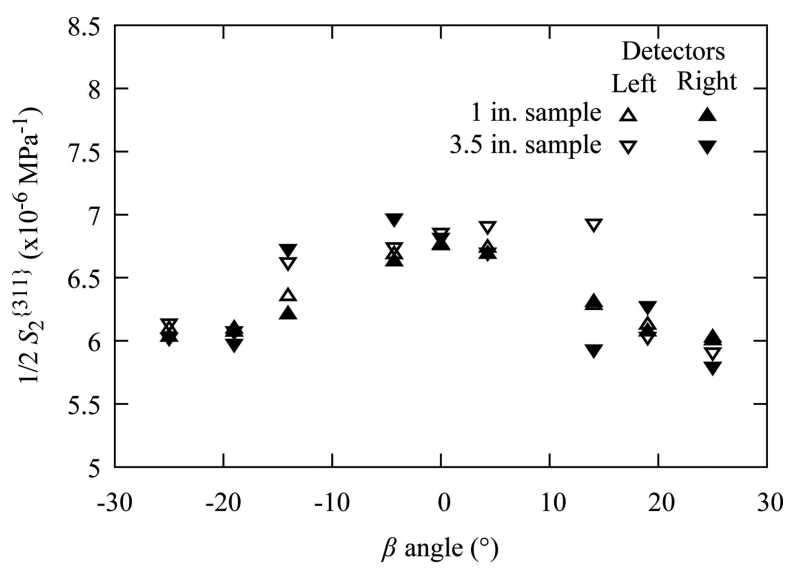

(a)

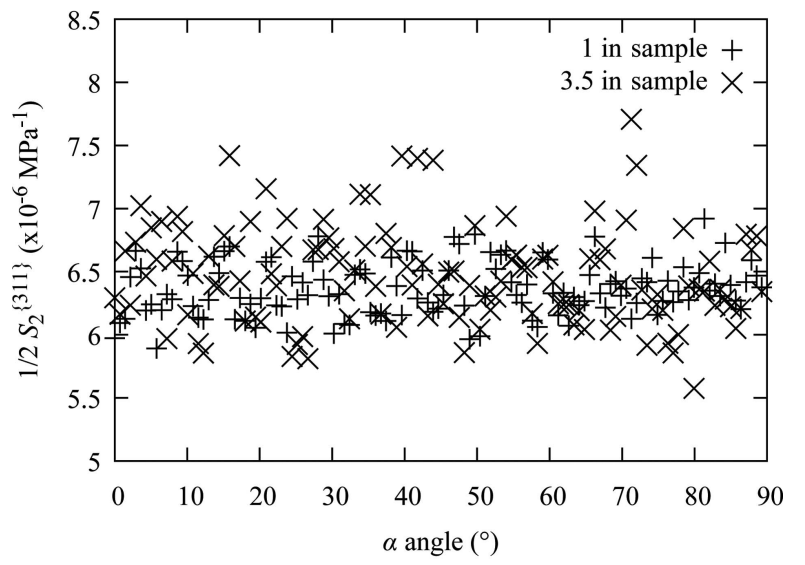

(b)

Figure 21

Comparison of the $\mathrm{XEC} \frac{1}{2} S_{2}^{\{311\}}$ calculated for the 1 and 3.5 in samples for (a) nine $\beta$ angles in the case of measurements with two linear detectors and (b) $125 \alpha$ angles for the 2D detector at $\beta=30^{\circ}$. 


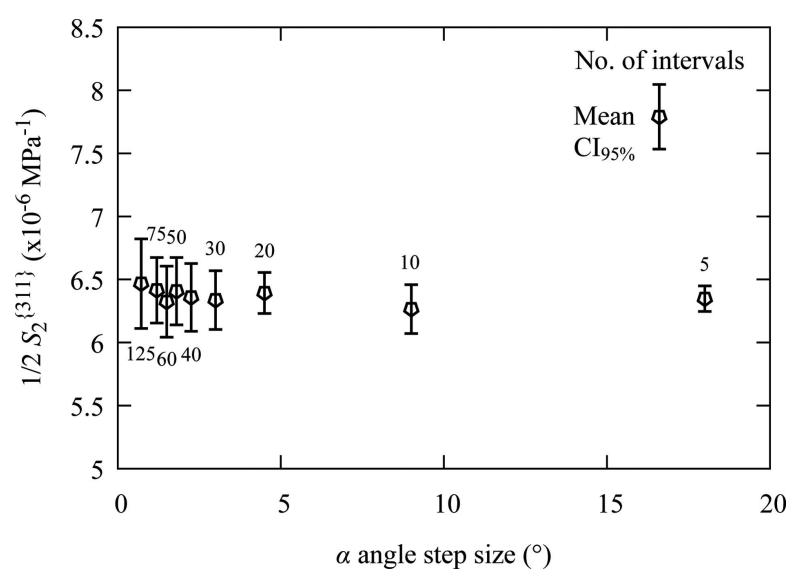

Figure 22

Mean and confidence interval $\left(\mathrm{CI}_{95 \%}\right)$ of the $\mathrm{XEC} \frac{1}{2} S_{2}^{\{311\}}$ calculated for different $\alpha$-step sizes for the 3.5 in sample. The corresponding number of $\alpha$ angles is presented below or above the error bar. $\mathrm{CI}_{95 \%}$ is the lower bound of the $95 \%$ confidence interval on the standard deviation.

The mean value is higher than that found for the 1 in sample and, more importantly, the confidence interval has broadened by $44 \%$.

The XEC was also calculated for the 2D detector at $\beta=30^{\circ}$ and for $125 \alpha$ angles. The XEC values computed for the 3.5 in sample are compared with those obtained for the 1 in sample in Fig. 21(b). The average value is also found to be higher than that of the 1 in sample value, $6.47 \times 10^{-6} \mathrm{MPa}^{-1}$, whereas the confidence interval of $0.36 \times 10^{-6} \mathrm{MPa}^{-1}$ has nearly doubled. Fig. 22 shows the XEC mean values for different $\alpha$-step sizes for comparison with Fig. 15(b). As for the 1 in sample, the confidence interval narrows when the step size increases, but remains significantly higher.

\section{Discussion}

X-ray diffraction techniques for residual stress measurements are based on the information coming from the distinct sets of grains satisfying the conditions for diffraction. Each strain used for the determination of the macro-stress is an average of the grain deformation in the set of diffracting grains. Because of the specific crystal elastic anisotropy of the grains in the measurement direction, the elastic behavior of the diffracting grains may differ from the macroscopic behavior (Noyan \& Cohen, 1987; Hauk, 1997). Consequently, the $\{h k l\}$-dependent XECs $S_{1}^{\{h k l\}}$ and $\frac{1}{2} S_{2}^{\{h k l\}}$ have to be carefully measured or computed. Sample texture is also known to play a major role in stress measurements and must be taken into account when computing the XEC (Hauk, 1997).

In the cases studied in this work, the two samples (homogeneous and bimodal microstructures) do not exhibit strong textures (Fig. 5). Nonetheless, both types of detector create some 'artificial' texture, which differs from one detector to the other owing to the diffraction conditions, as shown in Fig. 11. The 'artificial' textures determined for the homogeneous sample can be significant when considering a measurement at a given $\beta$ angle or $\alpha$ angle, for the linear detectors and the 2D detector, respectively (visible in Figs. 12 and 13). These textures result in some variations in the estimated XEC $\frac{1}{2} S_{2}^{\{311\}}$. Variations of up to $10 \%$ of the XEC value have been observed between the different sets of diffracting grains and the XEC value of the $\{311\}$ planes computed for the Hill bound in the case of an isotropic material. This value reaches $18 \%$ for the 3.5 in sample and its bimodal microstructure, which also plays a role in the diffraction measurement. Indeed, the lower number of grains reduces the number of orientations taken into consideration, resulting in a wider confidence interval on the standard deviation of the estimated XEC for both techniques.

It has been shown that increasing the grain size of a homogeneous microstructure leads to a significant broadening of the confidence interval on the standard deviation. Nonetheless, the percentage of grains contributing to the diffraction stays almost constant for both diffractometers (Fig. 18). For an average grain size of $90 \mu \mathrm{m}(R=1 / 15)$ and above, the default $0.72^{\circ} \alpha$-step size of the $2 \mathrm{D}$ detector is not sufficiently large to gather diffraction data in all regions of the Debye ring. Therefore the $\alpha$-step size had to be increased to $1.2^{\circ}$ to compute the XEC for all $\alpha$ steps.

The lack of diffraction data resulting from the diffraction texture or grain size can be overcome by the oscillation of the diffractometer around its $\beta$ position in the $\varphi$ plane (Fitzpatrick et al., 2005). The simulations have shown that adding a $2^{\circ}$ oscillation to the measurement increases the number of diffracting grains by up to $55 \%$ for the $2 \mathrm{D}$ detector and $36 \%$ for the linear detectors. Further increase of the number of diffracting grains is limited by the superimposition of the $\beta$ positions for the $\sin ^{2} \psi$ method and the linear detectors, as it is a multi-angle-based method. Indeed, increasing the oscillation angle to more than half the angle between two $\beta$ inclinations (Table 3) would lead to diffracting grains contributing to the diffraction data of two diffraction vector sets. This would mean that the diffraction data of a grain could be used for the measurement of two different strains, i.e. two different points in the plot $\varepsilon_{\varphi \psi}^{\{h k l\}}$ versus $\sin ^{2} \psi$, which may lead to inaccuracy in the linear regression between $\varepsilon_{\varphi \psi}^{\{h k l\}}$ and $\sin ^{2} \psi$, and therefore an inaccurate stress determination. In both cases, the oscillations narrow the confidence interval on the standard deviation of the estimated XEC, when compared with the intervals of the non-oscillating cases.

The low number of shared diffracting grains between the two methods (representing 20 and $14 \%$ of the total identified diffracting grains for the $\sin ^{2} \psi$ and $\cos \alpha$ methods, respectively) implies that the strains are measured from different sets of grains. These sets have different elastic behaviors, as observed in this study by the XEC variations, and may lead to the determination of different residual stresses.

Moreover, the residual stress measurement relies on the existence of a linear relationship between $\varepsilon_{\varphi \psi}^{\{h k l\}}$ or $\bar{\varepsilon}_{\alpha}^{\{h k l\}}$ and the $\sin ^{2} \psi$ or the $\cos \alpha$ values, respectively. Some authors (Hauk, 1997; Fitzpatrick et al., 2005) have shown that, when a low number of exposures are considered in the $\sin ^{2} \psi$ method, errors arise in the linear regression and the estimated residual stress values may be wrong. Even if the $\cos \alpha$ method 
generates more points for the linear regression, a compromise between the number of diffracting grains for a given point and the number of points considered for the linear regression (i.e. the $\alpha$-step size) should be found. In fact, for a small $\alpha$-step size and in the case of a large grain microstructure (low number of diffracting grains), statistical effects will result in large variations of the data $\bar{\varepsilon}_{\alpha}^{\{h k l\}}$ used to estimate the residual stress [equation (14)], leading to a significant error in the slope of the regression line used to compute the residual stress. Increasing the $\alpha$-step size will reduce the statistical errors but also reduce the number of points available for the linear regression. There is then an opportunity to develop further the current approach to better specify the optimum conditions that will minimize the errors when estimating an actual residual stress field. Moreover, the presence of some crystallographic texture in a material could be taken into consideration as it is known to result in additional difficulties in estimating the actual residual stresses in a component.

\section{Conclusions}

The objective of this study was to compare the traditional $\mathrm{X}$-ray diffraction technique for residual stress measurement, i.e. the $\sin ^{2} \psi$ method, and the recently implemented $\cos \alpha$ method through the elastic properties of the diffracting grains. For this purpose, a MATLAB script has been developed to identify the diffracting grains and two EBSD maps were used to generate realistic distributions of orientation and grain size. The specificities of the two XRD residual stress measurement methods were taken into consideration and various parameters were varied to better illustrate their effect on the quality of potential residual stress measurements. From the sets of identified diffracting grains, the following results have been observed:

(1) The $2 \mathrm{D}$ detector used by the $\cos \alpha$ method captures, in a single exposure, diffraction data from 1.5 times more unique diffracting grains than nine angular positions of the linear detectors used in the $\sin ^{2} \psi$ method (acceptable number of points allowing the determination of residual stresses via this method). Increasing the number of $\beta$ angles in the $\sin ^{2} \psi$ method does not allow the same number of unique diffracting grains to be reached. Keeping a realistic time of X-ray exposure for residual stress measurement purposes, nine exposures are proposed with the consequence that about $50 \%$ fewer unique grains are diffracting.

(2) The 'artificial' crystallographic texture effect related to the two XRD methods has been calculated. It was shown that the two methods yield two different types of textures. Furthermore, the diffracting grains detected by the linear detector method generate a higher texture index than for the $2 \mathrm{D}$ detector, even if the nine incident $\beta$ angles are used (about $45 \%$ higher). Nonetheless, when considering the stress calculation specificity of the $\cos \alpha$ method - division of the Debye ring into $125 \alpha$ angles - the highest texture index computed for the different $\alpha$ angles is similar to that found for the linear detectors at a given tilt angle ( $\beta$ angle). Artifacts can arise from the strong 'artificial' texture during residual stress computations.

(3) The detrimental effect of large grain size was simulated. It can be overcome by the oscillation of the diffractometer in the $\varphi$ plane. For the $\sin ^{2} \psi$ method, the oscillation angle should be carefully chosen so that it remains below half of the angle between two $\beta$ positions, otherwise a superimposition of the $\beta$ positions may occur, limiting the beneficial effect of this parameter. In addition to the oscillations, the $\cos \alpha$ method can use different $\alpha$-step sizes to overcome the grain-size effects.

The variations in XEC could lead to a nonlinear regression for the residual stress computation and therefore a large error in the slope determination, resulting in an inaccurate residual stress measurement.

Future work could include textured materials, such as rolled aluminium alloy plates, to study the effect of material texture on the 'artificial' XRD texture and on the XEC.

\section{Acknowledgements}

DD would like to thank Dr Sébastien Le Corre and Dr Benjamin Tressou for fruitful discussions.

\section{Funding information}

This work was financially supported by the Consortium of Research and Innovation in Aerospace in Quebec, the Natural Sciences and Engineering Research Council of Canada, Pratt \& Whitney Canada, Bell Helicopter Textron, L3-Communications MAS, Heroux Devtek, and Mathematics of Information Technology and Complex systems (grant No. RDC 435539-12).

\section{References}

Aba-Perea, P. E., Pirling, T., Withers, P. J., Kelleher, J., Kabra, S. \& Preuss, M. (2016). Mater. Des. 89, 856-863.

ASTM International (2012). Standard Test Method for Residual Stress Measurement by X-ray Diffraction for Bearing Steels. ASTM Standard E2860-12. ASTM International.

ASTM International (2013). E112-13 Standard Test Methods for Determining Average Grain Size. ASTM Standard E112-13. ASTM International.

Bachmann, F., Hielscher, R. \& Schaeben, H. (2011). Ultramicroscopy, 111, 1720-1733.

Cullity, B. D. (1956). Elements of X-ray Diffraction. Reading: Addison-Wesley Publishing Company.

Delbergue, D., Lévesque, M. \& Bocher, P. (2017). Proceedings of the 13th International Conference on Shot Peening, pp. 237-243. Montréal, QC, Canada. https://shotpeening.org/ICSP/icsp-13.php.

Delbergue, D., Texier, D., Lévesque, M. \& Bocher, P. (2016). Proceedings of the 10th International Conference on Residual Stresses 2016 (ICRS-10), edited by T. M. Holden, O. Muránsky \& L. Edwards, Materials Research Proceedings, Vol. 2, pp. 55-60. Millersville: Materials Research Forum.

Eatough, M. O., Rodriguez, M. A., Blanton, T. N. \& Tissot, R. G. (1997). Adv. X-ray Anal. 41, 319-326. 
Erinosho, T. O., Collins, D. M., Wilkinson, A. J., Todd, R. I. \& Dunne, F. P. E. (2016). Int. J. Plast. 83, 1-18.

Etter, A. L. \& Baudin, T. (2013). Rayonnement Synchrotron, Rayons $X$ et Neutrons au Service des Materériaux, edited by A. Lodini \& T. Baudin, ch. 6, pp. 278-321. Les Ulis: EDP Sciences.

Fitzpatrick, M., Fry, A., Holdway, P., Kandil, F., Shackleton, J. \& Suominen, L. (2005). Determination of Residual Stresses by $X$-raye Diffraction. National Physical Laboratory, Teddington, UK.

Frija, M., Hassine, T., Fathallah, R., Bouraoui, C. \& Dogui, A. (2006). Mater. Sci. Eng. A, 426, 173-180.

Gariépy, A., Bridier, F., Hoseini, M., Bocher, P., Perron, C. \& Lévesque, M. (2013). Surf. Coat. Technol. 219, 15-30.

Gnäupel-Herold, T., Brand, P. C. \& Prask, H. J. (1998). J. Appl. Cryst. 31, 929-935.

Guagliano, M. (2001). J. Mater. Process. Technol. 110, 277-286.

Haldipur, P. (2006). PhD thesis, Iowa State University, USA.

Hauk, V. (1997). Structural and Residual Stress Analysis by Nondestructive Methods: Evaluation, Application, Assessment. Amsterdam: Elsevier.

He, B. B. (2009). Two-Dimensional X-ray Diffraction. Hoboken: J. Wiley \& Sons.

Heydari Astaraee, A., Miresmaeili, R., Bagherifard, S., Guagliano, M. \& Aliofkhazraei, M. (2017). Mater. Des. 116, 365-373.

Hielscher, R. (2013). J. Multivariate Anal. 119, 119-143.

Hill, R. (1952). Proc. Phys. Soc. Sect. A, 65, 350-354.

Hines, W. W., Montgomery, D. C., Goldsman, D. M. \& Borror, C. M. (2003). Probability and Statistics in Engineering, 4th ed. Hoboken: John Wiley \& Sons.

Hiratsuka, K., Sasaki, T., Seki, K. \& Hirose, Y. (2003). Adv. X-ray Anal. 46, 61-67.

Hörz, F. \& Quaide, W. L. (1973). Moon, 6, 45-82.

Howard, C. J. (1982). J. Appl. Cryst. 15, 615-620.

Klotz, T., Delbergue, D., Bocher, P., Lévesque, M. \& Brochu, M. (2018). Int. J. Fatigue, 110, 10-21.

Klotz, T., Miao, H. Y., Bianchetti, C., Lévesque, M. \& Brochu, M. (2018). Int. J. Fatigue, 113, 204-221.

Kocks, U. F., Tomé, C. N. \& Wenk, H. R. (1998). Texture and Anisotropy: Preferred Orientations in Polycrystals and Their Effect on Materials Properties. Cambridge University Press.

Kohri, A., Takaku, Y. \& Nakashiro, M. (2016). Proceedings of the 10th International Conference on Residual Stresses 2016 (ICRS-10)\}, edited by T. M. Holden, O. Muránsky \& L. Edwards, Materials Research Proceedings, Vol. 2, pp. 103-108. Millersville: Materials Research Forum.

Ledbetter, H. M. \& Reed, R. P. (1973). J. Phys. Chem. Ref. Data, 2, 531-618.
Mainprice, D., Hielscher, R. \& Schaeben, H. (2011). Geol. Soc. London Spec. Publ. 360, 175-192.

Margetan, F., Nieters, E., Haldipur, P., Brasche, L., Chiou, T., Keller, M., Degtyar, A., Umbach, J., Hassan, W., Patton, T. \& Smith, K. (2005). Fundamental Studies of Nickel Billet Materials-Engine Titanium Consortium II. Technical Report. US Department of Transportation, Washington, DC, USA.

Miyazaki, T. \& Sasaki, T. (2016). J. Appl. Cryst. 49, 426-432.

Murray, C. E. (2013). J. Appl. Phys. 113, 153509.

Noyan, I. C. \& Cohen, J. B. (1987). Residual Stress Measurement by Diffraction and Interpretation. New York: Springer-Verlag.

Peterson, N., Kobayashi, Y. \& Sanders, P. (2017). Proceedings of the 13th International Conference on Shot Peening, pp. 80-86. Montréal, QC, Canada. https://shotpeening.org/ICSP/icsp-13.php.

Prevéy, P. S. (1986). Materials Characterization, Vol. 10, pp. 380-392. Materials Park: ASM International.

Prevéy, P. S. (2000). Proceedings of the 20th ASM Materials Solution Conference and Exposition. St Louis, Missouri, USA.

Ramirez-Rico, J., Lee, S.-Y., Ling, J. J. \& Noyan, I. C. (2016). J. Mater. Sci. 51, 5343-5355.

Ruud, C. (2002). Handbook of Residual Stress and Deformation of Steel, edited by G. Totten, M. Howes \& T. Inoue, pp. 99-117. Materials Park: ASM International.

SAE International (2003). Residual Stress Measurement by X-ray Diffraction. Standard SAE HS784. SAE International.

SAE International (2009). Nickel Alloy, Corrosion and HeatResistant, Bars, Forgings, and Rings 52.5Ni-19Cr-3.0Mo-5.1Cb (Nb)-0.90Ti-0.50Al-18Fe Consumable Electrode or Vacuum Induction Melted $1775^{\circ} \mathrm{F}\left(968^{\circ} \mathrm{C}\right)$ Solution and Precipitation Heat Treated. Standard SAE AMS5663M. SAE International.

Sasaki, T. (2014). Mater. Sci. Forum, 783-786, 2103-2108.

Sasaki, T., Hirose, Y., Sasaki, K. \& Yasukawa, S. (1997). Adv. X-ray Anal. 40, 588-594

Schajer, G. S. (2013). Practical Residual Stress Measurement Methods. Chichester: Wiley.

Taira, S., Tanaka, K. \& Yamasaki, T. (1978). J. Soc. Mater. Sci. Jpn, 27, 251-256.

Tanaka, K. (2018). J. Appl. Cryst. 51, 1329-1338.

Tu, F., Delbergue, D., Klotz, T., Bag, A., Miao, H. Y., Bianchetti, C., Brochu, M., Bocher, P. \& Levesque, M. (2018). Int. J. Mech. Sci. 145, 353-366.

Tu, F., Delbergue, D., Miao, H. Y., Klotz, T., Brochu, M., Bocher, P. \& Lévesque, M. (2017). Surface Coatings Technol. 319, 200-212.

Van Houtte, P. \& De Buyser, L. (1993). Acta Metall. Mater. 41, $323-$ 336.

Webster, G. A. \& Ezeilo, A. N. (2001). Int. J. Fatigue, 23, 375-383. 